\title{
Sub-GeV Dark Matter as Pseudo-Nambu-Goldstone Bosons from the Seesaw Scale
}

\author{
Michele Frigerio, ${ }^{1,2,3, *}$ Thomas Hambye, ${ }^{4, \dagger}$ and Eduard Masso ${ }^{5}$ \\ ${ }^{1}$ CNRS, Laboratoire Charles Coulomb, UMR 5221, F-34095 Montpellier, France, \\ ${ }^{2}$ Université Montpellier 2, Laboratoire Charles Coulomb, UMR 5221, F-34095 Montpellier, France \\ ${ }^{3}$ Institut de Física d'Altes Energies, Universitat Autònoma de Barcelona, E-08193 Bellaterra, Spain \\ ${ }^{4}$ Service de Physique Théorique, Université Libre de Bruxelles, 1050 Bruxelles, Belgium \\ ${ }^{5}$ Grup de Física Teòrica, Departament de Física, Universitat Autònoma de Barcelona, E-08193 Bellaterra, Spain
}

(Received 5 August 2011; published 29 December 2011)

\begin{abstract}
Pseudo-Nambu-Goldstone bosons (pNGBs) are naturally light, spin-zero particles that can be interesting dark-matter (DM) candidates. We study the phenomenology of a pNGB $\theta$ associated with an approximate symmetry of the neutrino seesaw sector. A small coupling of $\theta$ to the Higgs boson is induced radiatively by the neutrino Yukawa couplings. By virtue of this Higgs-portal interaction, (i) the pNGB acquires a mass $m_{\theta}$ proportional to the electroweak scale, and (ii) the observed DM relic density can be generated by the freeze-in of $\theta$ particles with mass $m_{\theta} \simeq 3 \mathrm{MeV}$. Alternatively, the coupling of $\theta$ to heavy sterile neutrinos can account for the DM relic density, in the window $1 \mathrm{keV} \lesssim m_{\theta} \lesssim 3 \mathrm{MeV}$. The decays of $\theta$ into light fermions are suppressed by the seesaw scale, making such pNGBs sufficiently stable to play the role of DM.
\end{abstract}

DOI: 10.1103/PhysRevX.1.021026

Subject Areas: Particles and Fields

\section{INTRODUCTION}

The astrophysical and cosmological evidence for dark matter (DM) requires that a new particle be introduced, with constrained mass and interactions. These interactions must, in particular, maintain the DM candidate as stable on cosmological time scales and generate the observed DM relic abundance. Many candidates can satisfy all constraints. Much fewer, though, provide a convincing explanation for the required values of the DM parameters. As examples, in most models, (i) an ad-hoc symmetry is postulated to insure stability, and/or (ii) the DM mass is assumed to be of order (or below) the electroweak (EW) scale as demanded by the weakly interacting massive particle (WIMP) paradigm, and/or (iii) new interactions with strength similar to the EW ones, not motivated by other means, are just postulated. A scenario which is potentially predictive, and could provide motivations for some of these assumptions, consists in identifying the DM candidate with the pseudo-Nambu-Goldstone boson (pNGB) of a spontaneously broken global symmetry.

An interesting feature of such a scenario is that the DM couplings to the standard-model (SM) fields, which could render the DM candidate unstable over cosmological times, are suppressed by powers of the spontaneoussymmetry-breaking (SSB) scale. As a result, provided this scale is large, the decay width of the particle can be

\footnotetext{
*frigerio@ifae.es

†thambye@ulb.ac.be
}

Published by the American Physical Society under the terms of the Creative Commons Attribution 3.0 License. Further distribution of this work must maintain attribution to the author(s) and the published article's title, journal citation, and DOI. sufficiently small. A well-known example is the axion, the pNGB that is associated with the Peccei-Quinn $U(1)$ symmetry, broken spontaneously at the scale $f_{\mathrm{PQ}}$. The axion is a viable DM candidate when $f_{\mathrm{PQ}}$ lies in the interval of approximately $\left(10^{12}-10^{14}\right) \mathrm{GeV}$. Another possibility is to consider SSB at the seesaw scale, which should be large to account for the small size of the neutrino masses. One global symmetry related to the seesaw scale is $U(1)_{B-L}$. In the case that it is spontaneously broken by an EW singlet, coupled to heavy-sterile- (right-handed-) neutrinos, the corresponding NGB is known as the singlet Majoron [1]. Models with a pseudo-Majoron as DM have been studied [2-7]. More generally, we will consider any (family-dependent) global symmetry whose spontaneous breaking contributes to sterile-neutrino masses. Symmetries associated with the seesaw sector have the advantage that the pNGB interactions are anticipated to exist anyway to account for neutrino masses.

Another remarkable property of the pNGB scenario, relevant for the DM phenomenology, is that the mass and the scalar-potential interactions of a pNGB vanish in the limit of exact symmetry. Therefore, when the symmetry is explicitly broken by a unique (or dominant) term, the scalar interactions and the DM mass are necessarily related, and, if the relic density is essentially determined by such interactions, this implies a one-to-one correspondence between the DM relic density and the DM mass. For instance, we will consider a framework in which the source of explicit breaking generates radiatively a "Higgs-portal" interaction of the form $\lambda \theta^{2} H^{\dagger} H / 2$, where $\theta$ is the pNGB particle and $H$ is the SM Higgs doublet. This coupling generates a mass $m_{\theta}^{2}=\lambda v^{2}$ with $v=174 \mathrm{GeV}$ and, at the same time, a relic density from the $\theta-H$ interactions. We will show how, given the known thermal distribution of SM 
particles in the early-Universe thermal bath, the Higgs portal can generate a DM relic density through the freeze-in mechanism. (See, e.g., Ref. [8].) The observed relic density is obtained for a unique possible value of the DM mass, which turns out to be $m_{\theta} \simeq 2.8 \mathrm{MeV}$ for a Higgs boson mass of $120 \mathrm{GeV}$. There is also a second possibility for generating the correct relic density: by the freeze-in of the interaction of $\theta$ with the sterile neutrinos. These production mechanisms differ from those previously considered for a pNGB candidate for DM.

A third interesting feature of the pNGB setup is that the DM mass is related to the scale of explicit symmetry breaking, which can be identified with a physical-mass scale already present in the theory, analogous to the QCD scale for the axion. For instance, in the scenario we will consider below, the neutrino Dirac Yukawa couplings provide the source of explicit breaking of the global symmetry. Therefore, the DM mass is proportional to the EW scale, $m_{\theta}^{2}=\lambda v^{2}$, and this provides a justification for the presence of a scalar DM particle at or below the EW scale. Moreover, the coupling $\lambda$ can be computed as a function of the seesaw couplings. In turn, we will show that, in this framework, the DM mass can be protected from quadratically divergent corrections. The mechanism we invoke to remove these corrections is a variation of the one proposed long ago by Hill and Ross for very light pNGBs with both scalar and pseudoscalar couplings [9]. We will consider a $U(1)_{X}$ symmetry such that its explicit breaking requires several Yukawa couplings at the same time, thus lowering the degree of divergence of the contributions to the pNGB effective potential. This mechanism has similarities with the collective-breaking mechanism introduced to protect the EW scale in little Higgs models, where the SM Higgs is the pNGB. (See Ref. [10] for a review.)

All in all, we will show that the seesaw interactions (i) are associated with global symmetries broken spontaneously at the heavy-neutrino-mass scale, whose large size guarantees the stability of the pNGB-DM candidate, and (ii) have a source of explicit symmetry breaking built in, and therefore they induce the mass and scalar interactions of the DM. Moreover, they can lead to (iii) a one-to-one correspondence between the DM mass and the DM relic density, with (iv) a justification for the presence of DM at low scale, and with (v) a DM mass protected from large radiative corrections.

The paper is organized as follows. In Sec. II, we display the relevant effective interactions of our pNGB candidate for DM. In Sec. III, we show how a pNGB relic density of the order of the observed DM density can be obtained through freeze-in. In Sec. IV, we present a class of seesaw models in which the required pNGB interactions are generated naturally. In Sec. V, we compute the pNGB couplings to SM particles and analyze the corresponding constraints on the DM stability. We conclude in Sec. VI.

\section{EFFECTIVE INTERACTIONS OF THE PNGB}

In this section, we introduce the effective Lagrangian of a pNGB $\theta$, associated with a global symmetry of the neutrino sector, broken spontaneously at the seesaw scale $f$. We postpone to Sec. IV the detailed description of a class of models where such a light pNGB may emerge naturally. For the present purposes, it suffices to specify the effective interactions of $\theta$ at low energy (below $f$ ).

In the class of models under consideration, $\theta$ couples to a sterile neutrino $\nu^{c}$ as follows:

$$
\begin{aligned}
\mathcal{L}_{N} & =-\frac{g f}{2 \sqrt{2}} \nu^{c} \nu^{c} e^{i \theta / f}+\text { H.c. } \\
& =-\frac{m_{N}}{2} \bar{N} N+\frac{i g}{2 \sqrt{2}} \theta \bar{N} \gamma_{5} N+\frac{g}{4 \sqrt{2} f} \theta^{2} \bar{N} N+\ldots,
\end{aligned}
$$

where $g$ is a Yukawa coupling, and the four-component Majorana neutrino is defined as usual by $N \equiv\left(\nu^{c} \nu^{c \dagger}\right)^{T}$. For simplicity, we consider only one sterile neutrino, whose mass $m_{N}=g f / \sqrt{2}$ is generated by SSB. In this case, the Lagrangian shown in Eq. (1) is the same as for the singlet Majoron model [1], where the spontaneously broken global symmetry is lepton number. However, we will show in Sec. IV that, in realistic cases with two (or more) sterile neutrinos, the relevant symmetry is not the ordinary lepton number; rather, each sterile neutrino carries a different charge, and consequently the pNGB $\theta$ cannot be identified with the Majoron. We remark that the $\theta-N$ interaction is just an example of a NGB interaction with a heavy fermion charged under the associated global symmetry. As a consequence, the implications for dark-matter phenomenology, discussed in the following sections, hold qualitatively in a more general set of theories, with heavy fermions other than the sterile neutrinos.

A nonvanishing potential for the NGB is generated when one introduces a source of explicit breaking of the global symmetry. In the models we will consider, this source is given by a certain set of neutrino Dirac Yukawa couplings, so that the $\theta$ effective potential necessarily involves the SM Higgs doublet $H$. The relevant, radiatively induced coupling is given by

$$
\mathcal{L}_{H}=-\frac{\lambda}{2} \theta^{2} H^{\dagger} H=-\frac{m_{\theta}^{2}}{2} \theta^{2}-\frac{\lambda v}{\sqrt{2}} \theta^{2} h-\frac{\lambda}{4} \theta^{2} h^{2},
$$

where $v \simeq 174 \mathrm{GeV}$ is the Higgs vacuum expectation value (VEV), $h$ is the physical Higgs boson, and we adopt the unitary gauge. Note that the mass of the pNGB $\theta$ is proportional to the EW VEV, $m_{\theta}^{2}=\lambda v^{2}$. This relation is sometimes referred to as the "conformal" limit, since $\theta$ has no bare-mass term; rather, the Higgs-portal interaction $\lambda$ generates both $m_{\theta}$ and the $\theta-h$ couplings. We remark that the phenomenology (e.g., the DM mass, its relic density, etc.) induced by the Higgs portal in Eq. (2) does not depend on the details of the underlying physics which generates $\lambda$ radiatively. 
In the specific seesaw models that we will build, $\lambda$ is generated by logarithmically divergent neutrino loops, involving both the $\theta-N$ coupling $g$ as well as neutrino Dirac Yukawa couplings, denoted generically by $y$. It turns out that the relation between the mass of $\theta$ and the neutrino parameters can be written schematically as

$$
m_{\theta}^{2}=\lambda v^{2} \simeq g^{2} y^{2} v^{2} \frac{\log \left(\Lambda^{2} / m_{N}^{2}\right)}{8 \pi^{2}},
$$

where $\Lambda$ is some cutoff scale at or above $f$. The lightneutrino-mass scale is given by the standard seesaw relation, $m_{\nu}=y^{2} v^{2} / m_{N}$. It is useful to use Eq. (3) to express the coupling $g$ as a function of the relevant energy scales in the theory:

$$
g=10^{-3}\left(\frac{m_{\theta}}{\mathrm{MeV}}\right)\left(\frac{\mathrm{eV}}{m_{\nu}}\right)^{1 / 2}\left(\frac{10^{9} \mathrm{GeV}}{m_{N}}\right)^{1 / 2}\left(\frac{8 \pi^{2}}{\log \left(\Lambda^{2} / m_{N}^{2}\right)}\right)^{1 / 2} .
$$

We will take the factor $k \equiv \log \left(\Lambda^{2} / m_{N}^{2}\right) /\left(8 \pi^{2}\right)$ to be of order 1. Then, for a fixed value of $m_{\nu}, m_{N}$ and also $f=$ $\sqrt{2} m_{N} / g$ are determined as a function of $m_{\theta}$ and $g$, which are basically the only two free parameters. We require the scale $f$ to be smaller than the Planck scale, $M_{\mathrm{P}}=1.22 \times$ $10^{19} \mathrm{GeV}$. In addition, we assume for simplicity that $f$ is larger than $1 \mathrm{TeV}$, in order for the effective theory to contain only the SM particles, the pNGB, and the sterile

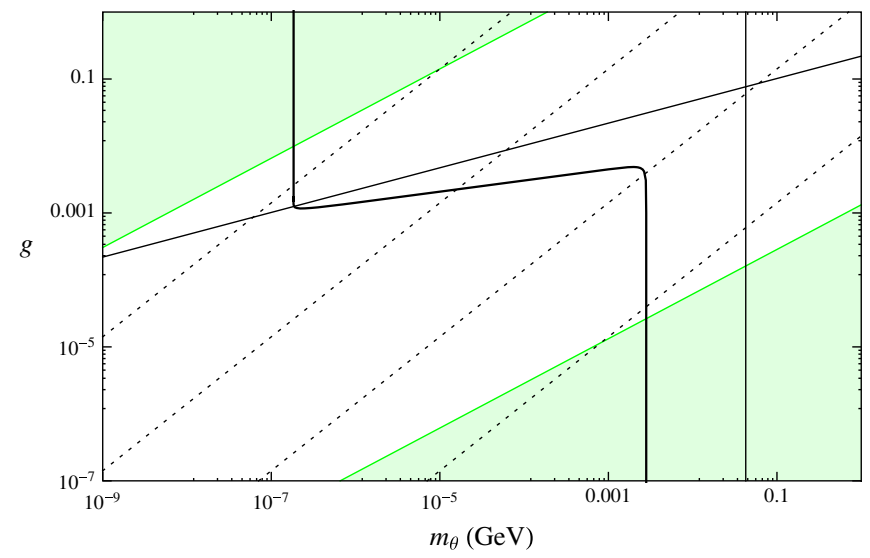

FIG. 1. The pNGB coupling to the sterile neutrino, $g$, versus the pNGB mass, $m_{\theta}$, in GeV. We have fixed $m_{\nu}=0.05 \mathrm{eV}$ and $k=1$. The upper (lower) green shaded region is excluded by requiring the SSB scale $f$ to be larger than $1 \mathrm{TeV}$ (smaller than the Planck scale). The four dotted lines correspond to these constant values of the sterile-neutrino mass, from top to bottom: $m_{N}=10^{2}, 10^{6}, 10^{10}, 10^{14} \mathrm{GeV}$. The thin diagonal (vertical) line indicates the lower value of $g\left(m_{\theta}\right)$ that leads to a thermalization of $\theta$. The thick curving line corresponds to a $\theta$ relic density equal to the observed DM relic density, as follows from the numerical solution of the Boltzmann equation (for $m_{h}=120 \mathrm{GeV}$ ). Since $\theta$ is produced by freeze-in, its relic density grows with its couplings $g$ and $\lambda=m_{\theta}^{2} / v^{2}$; therefore, the region below and to the left (above and to the right) of the thick curving line corresponds to $\Omega_{\theta}<\Omega_{\mathrm{DM}}\left(>\Omega_{\mathrm{DM}}\right)$. neutrino, while the degrees of freedom involved in the SSB are decoupled. These two requirements exclude the greenshaded regions in the $m_{\theta}-g$ plane shown in Fig. 1. The dotted lines in Fig. 1 correspond to constant values of the sterile-neutrino mass, $m_{N}=m_{\theta}^{2} /\left(g^{2} m_{\nu} k\right)=10^{2}, 10^{6}$, $10^{10}, 10^{14} \mathrm{GeV}$, from top to bottom.

\section{RELIC DENSITY FROM FREEZE-IN OF THE PNGB}

We now study the role of the interactions in Eqs. (1) and (2) for the production of a $\theta$ relic density, which can play the role of DM. In this section, we implicitly assume that $\theta$ is stable on cosmological time scales. The region of parameters where this stability is achieved will be analyzed in detail in Sec. V. There are several mechanisms that can produce the DM relic density. We begin by briefly recalling these various possibilities and confronting them with the parameter space of our scenario.

A well-known way to produce a cosmological density of a DM particle species is the usual freeze-out: It requires an annihilation cross section large enough to overcome the expansion of the Universe and thermalize the DM particle. The interactions of $\theta$ come from the terms in Eq. (1), which we refer to as the "sterile-neutrino portal", and the terms in Eq. (2), that is, the "Higgs portal". These interactions may or may not lead to the thermalization of $\theta$ in the early Universe, depending on whether the interaction rate $\Gamma$ gets larger than the Hubble rate $H$. If it does, later on, $\theta$ necessarily decouples from the plasma and, if stable, acts as DM with a certain energy density $\rho_{\theta}$, which depends in general on its mass and annihilation cross section.

Before determining $\rho_{\theta}$ numerically, it is instructive to identify the thermalization region in first approximation by evaluating the $\theta$ interaction rate $\Gamma$ and requiring $\Gamma>H$. We start with the Higgs portal, considering for simplicity the $h \rightarrow \theta \theta$ decay only, because, in our scenario, decays turn out to dominate with respect to scattering processes. (See Fig. 2.) The decay rate is

$$
\Gamma(h \rightarrow \theta \theta)=\frac{1}{16 \pi} \lambda^{2} \frac{v^{2}}{m_{h}} \sqrt{1-\frac{4 m_{\theta}^{2}}{m_{h}^{2}}} .
$$

This should be compared to the Hubble parameter in the radiation epoch, which is given by $H=1.66 \sqrt{g_{*}^{\rho}} T^{2} / M_{P}$, where $g_{*}^{\rho}=106.75$ is the degrees of freedom corresponding to the SM content. (We shall assume such value in the rest of the paper.) Taking $T \simeq m_{h}$ to be the relevant temperature, we find that, for a coupling

$$
\lambda \geq 6 \times 10^{-8}\left(\frac{m_{h}}{120 \mathrm{GeV}}\right)^{3 / 2},
$$

the decays and inverse decays are able to produce a thermal population of $\theta$ particles. In other words, given the $m_{\theta}^{2}=$ $\lambda v^{2}$ relation, one needs $m_{\theta}>44 \mathrm{MeV}$, which translates into the thin vertical line in Fig. 1 [11]. 


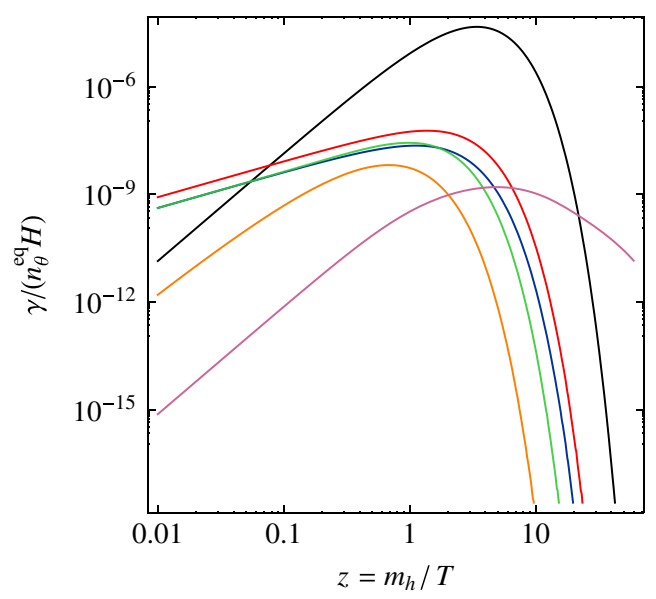

FIG. 2. The decay thermalization rate, $\gamma_{D h} /\left(n_{\theta}^{\mathrm{eq}} H\right)$ (black curve), compared to the various scattering thermalization rates, $\gamma_{\text {annih }}^{a} /\left(n_{\theta}^{\mathrm{eq}} H\right)$ for $a=W, Z, h, t, b$ ( the red, blue, green, orange and purple curves, respectively), as a function of $z=m_{h} / T$ and for $m_{h}=120 \mathrm{GeV}, m_{\theta}=2.8 \mathrm{MeV}$. For freeze-in these rates remain always well below 1 , which is the thermalization value.

Similarly, for the interaction with the sterile neutrino $N$, the process $N N \rightarrow \theta \theta$ has a rate

$$
\Gamma=\left\langle\sigma v_{\text {rel }}\right\rangle n_{N} \simeq \frac{g^{4}}{256 \pi} \frac{n_{N}}{m_{N}^{2}} \simeq 2 \times 10^{-4} g^{4} m_{N},
$$

where $n_{N}$ is the sterile-neutrino number density, and we used $T \simeq m_{N}$. This leads to thermal production of $\theta$ particles, provided that

$$
g \gtrless 10^{-2}\left(\frac{\mathrm{eV}}{m_{\nu} k}\right)^{1 / 6}\left(\frac{m_{\theta}}{\mathrm{MeV}}\right)^{1 / 3},
$$

where we made use of Eq. (4). This gives the thin diagonal line in Fig. 1. We do not discuss the effects of the couplings of $\theta$ to light fermions because, in the models under consideration, they turn out to be far too small to thermalize $\theta$. (See Sec. V.)

Outside the thermalization region, where $\Gamma$ is smaller than $H$, one may still have a final $\Omega_{\theta}$ matching $\Omega_{\mathrm{DM}}$. (As usual, we define $\Omega_{i} \equiv \rho_{i} / \rho_{\text {crit }}$, i.e., the ratio between the energy density $\rho_{i}$ and the critical density of the Universe.) The idea is that in the early Universe a population of $\theta$ particles is produced through pair-production-scattering processes, but with a density smaller than the thermal one. For appropriate values of $m_{\theta}$, one may still reach a relic density equal to $\Omega_{\mathrm{DM}}$. This process, called freeze-in, actually happens in our scenario, as we shall describe below in detail. (For generic properties of the freeze-in mechanism, see Ref. [8].)

In the rest of this section, we first introduce the ingredients to make a numerical calculation of the $\theta$ relic density, and we then apply them to the freeze-out and freeze-in processes. Afterward, we also discuss some nonthermal production mechanisms, and we end with a summary of our results.

\section{A. Boltzmann equation and reaction densities}

From the Higgs portal, $\theta$ particles are created from the following scattering processes [12]:

$$
\begin{gathered}
\sigma(h h \rightarrow \theta \theta) \simeq \frac{\lambda^{2}}{32 \pi s}\left(\frac{s-4 m_{\theta}^{2}}{s-4 m_{h}^{2}}\right)^{1 / 2}\left(\frac{s+2 m_{h}^{2}}{s-m_{h}^{2}}\right)^{2} \\
\sigma(W W \rightarrow \theta \theta)=\frac{1}{9} \frac{\lambda^{2}}{32 \pi s}\left(\frac{s-4 m_{\theta}^{2}}{s-4 m_{W}^{2}}\right)^{1 / 2} \frac{s^{2}-4 s m_{W}^{2}+12 m_{W}^{4}}{\left(s-m_{h}^{2}\right)^{2}+m_{h}^{2} \Gamma_{h}^{2}} \\
\sigma(Z Z \rightarrow \theta \theta)=\frac{1}{9} \frac{\lambda^{2}}{32 \pi s}\left(\frac{s-4 m_{\theta}^{2}}{s-4 m_{Z}^{2}}\right)^{1 / 2} \frac{s^{2}-4 s m_{Z}^{2}+12 m_{Z}^{4}}{\left(s-m_{h}^{2}\right)^{2}+m_{h}^{2} \Gamma_{h}^{2}}
\end{gathered}
$$

$$
\sigma(f \bar{f} \rightarrow \theta \theta)=\frac{1}{4} \frac{1}{n_{\mathrm{c}}} \frac{\lambda^{2}}{16 \pi s} \frac{m_{f}^{2}\left(s-4 m_{f}^{2}\right)^{1 / 2}\left(s-4 m_{\theta}^{2}\right)^{1 / 2}}{\left(s-m_{h}^{2}\right)^{2}+m_{h}^{2} \Gamma_{h}^{2}},
$$

where $\Gamma_{h}$ is the total Higgs decay width and $n_{\mathrm{c}}$ is the number of colors of the fermion $f$.

Similarly, $\theta$ particles can be created through the scattering with a sterile neutrino,

$$
\sigma(N N \rightarrow \theta \theta) \simeq \frac{g^{4}}{256 \pi} \frac{1}{\beta_{N}^{2} s}\left(\beta_{N} \frac{s}{m_{N}^{2}}+2 \log \frac{1-\beta_{N}}{1+\beta_{N}}\right),
$$

where we neglected $m_{\theta}$ in front of $m_{N}$ and $\sqrt{s}$, and we defined $\beta_{N}=\left(1-4 m_{N}^{2} / s\right)^{1 / 2}$.

In the following discussion, it will be convenient to consider separately the effect of the Higgs decay, Eq. (5), which means that, in order to avoid double counting, the on-shell part of the scattering processes must be subtracted (whenever there is one). In the narrow width approximation, this means one must perform in Eqs. (10)-(12) the substitution

$$
\begin{aligned}
\frac{m_{h} \Gamma_{h}}{\left(s-m_{h}^{2}\right)^{2}+m_{h}^{2} \Gamma_{h}^{2}} & \rightarrow \frac{m_{h} \Gamma_{h}}{\left(s-m_{h}^{2}\right)^{2}+m_{h}^{2} \Gamma_{h}^{2}} \\
& -\pi \delta\left(s-m_{h}^{2}\right) \theta\left(s-4 m_{i}^{2}\right),
\end{aligned}
$$

for $i=W, Z, f$. In the following discussion, all scattering quantities we will consider are meant to be the subtracted ones, using Eq. (14). To calculate the relic density that one obtains from these processes, either through freeze-in or freeze-out, one has to integrate the Boltzmann equation,

$z H(z) s(z) Y_{\theta}^{\prime}(z)=\left[1-\left(\frac{Y_{\theta}(z)}{Y_{\theta}^{\mathrm{eq}}(z)}\right)^{2}\right]\left(\gamma_{D_{h}}(z)+\gamma_{\mathrm{annih}}(z)\right)$,

where $z \equiv m_{h} / T$ is conventionally taken as the evolution parameter. Here, $H(z)$ is the Hubble parameter, $s(z)$ is the entropy density, and $Y_{\theta} \equiv n_{\theta} / s$, with $n_{\theta}$ the number density. The reaction density $\gamma_{D_{h}}$ contains the effect of the Higgs boson decay, while $\gamma_{\text {annin }}$ is the sum of the (subtracted) reaction densities of the scattering processes in Eqs. (9)(13). The reaction densities are given by 


$$
\begin{gathered}
\gamma_{D_{h}}=N_{\theta} \int d \bar{p}_{h} f_{h}^{\mathrm{eq}} \iint d \bar{p}_{1} d \bar{p}_{2}(2 \pi)^{4} \delta^{4}\left(p_{h}-p_{1}-p_{2}\right)|\mathcal{M}|^{2} \\
=N_{\theta} n_{h}^{\mathrm{eq}} \frac{K_{1}(z)}{K_{2}(z)} \Gamma(h \rightarrow \theta \theta) \\
\gamma(a b \leftrightarrow 12)=N_{\theta} \iint d \bar{p}_{a} d \bar{p}_{b} f_{a}^{\mathrm{eq}} f_{b}^{\mathrm{eq}} d \bar{p}_{1} \\
\quad \times \iint d \bar{p}_{2}(2 \pi)^{4} \delta^{4}\left(p_{a}+p_{b}-p_{1}-p_{2}\right)|\mathcal{M}|^{2} \\
=N_{\theta} \frac{T}{64 \pi^{4}} \int_{s_{\min }}^{\infty} d s \sqrt{s} \hat{\sigma}(s) K_{1}\left(\frac{\sqrt{s}}{T}\right)
\end{gathered}
$$

involving the Bessel functions $K_{1,2}$. We have defined $d \bar{p} \equiv$ $d^{3} p /\left((2 \pi)^{3} 2 E\right)$. Here, $N_{\theta}=2$ is the number of $\theta$ particles produced per decay or annihilation, $f_{i}^{\mathrm{eq}}=\left(e^{E_{i} / T} \pm 1\right)^{-1} \simeq$ $e^{-E_{i} / T}$ is the Maxwell-Boltzmann energy distribution, $|\mathcal{M}|^{2}$ is the amplitude squared summed over the initial and final spins (with no averaging), $s_{\min }=\max \left[\left(m_{a}+m_{b}\right)^{2}\right.$, $\left.\left(m_{1}+m_{2}\right)^{2}\right]$, and the reduced cross section is defined by

$\hat{\sigma}(a b \leftrightarrow 12)=\frac{g_{a} g_{b}}{c_{a b}} \frac{2\left[\left(s-m_{a}^{2}-m_{b}^{2}\right)^{2}-4 m_{a}^{2} m_{b}^{2}\right]}{s} \sigma(a b \rightarrow 12)$,

where $\sigma$ is the particle physics cross section of Eqs. (9)-(13), $g_{a, b}$ is the number of degrees of freedom of the particles $a, b$, and $c_{a b}$ is a combinatorial factor equal to 2 (1) if $a$ and $b$ are identical (different).

\section{B. Freeze-out}

As is well known, if $\theta$ freezes out relativistically, that is, at $T \geqslant m_{\theta}$, its relic density is independent of the annihilation cross section, because it decouples when the thermal number density, $Y_{\theta}^{\mathrm{eq}} \equiv n_{\theta}^{\mathrm{eq}} / s$, is still independent of the temperature. The formula for the relative density $\Omega_{\theta}$ is simply

$$
\Omega_{\theta} h_{0}^{2} \simeq 78 \frac{1}{g_{*}^{s}} \frac{m_{\theta}}{\mathrm{keV}},
$$

where $g_{*}^{s}$ is the number of degrees of freedom contributing to entropy at $\theta$ decoupling. This should match the value

$$
\Omega_{\mathrm{DM}} h_{0}^{2}=0.11 \pm 0.01 .
$$

In these equations, $h_{0}$ is the reduced Hubble constant $h_{0}=$ $H_{0} /\left(100 \mathrm{Mpc} \mathrm{km} \mathrm{s}^{-1}\right) \simeq 0.70$. As a result, the observed relic density can be obtained only for one DM mass value: $m_{\theta} \simeq 0.15 \mathrm{keV}$, where we took $g_{*}^{s}=106.75$, valid for a decoupling temperature above $100 \mathrm{GeV}$. Such a light $\theta$ can be only thermalized by the interaction with sterile neutrinos, which decouple at a temperature just below $m_{N}$, leading to relativistic $\theta$ 's. This result corresponds to the left vertical branch of the thick curve in Fig. 1, which is obtained by numerically integrating the Boltzmann equation.
If, instead, $\theta$ is heavier and thermalizes, it must decouple nonrelativistically in order to have a small enough relic density. This decoupling can occur through the Higgsportal interaction. In this case, the relic density essentially depends only on the annihilation cross section, which must take the value $\left\langle\sigma v_{\text {rel }}\right\rangle \simeq 10^{-26} \mathrm{~cm}^{3} \mathrm{~s}^{-1}$. To get a sufficiently small relic density, one needs to go to much higher values of $m_{\theta}$, where the coupling $\lambda=m_{\theta}^{2} / v^{2}$ becomes large enough. The observed relic density is obtained for a unique value of $m_{\theta}$ because of the relation between $m_{\theta}$ and $\lambda$, which is a consequence of the pNGB nature of $\theta$. The freeze-out through the Higgs portal was considered in Ref. [14], assuming this restrictive relation, and it was found that the DM relic density is obtained with $m_{\theta}=$ 50-70 GeV (for $m_{h}=120-180 \mathrm{GeV}$ ).

However, as we will see in Sec. V, the freeze-out values $m_{\theta}=0.15 \mathrm{keV}$ as well as $m_{\theta}=50-70 \mathrm{GeV}$ require values of parameters that are excluded by the instability of $\theta$ on cosmological time scales. Therefore, in our scenario, both relativistic and nonrelativistic freeze-out is not a viable way to produce DM. Instead, the freeze-in process turns out to be efficient in an allowed region of the parameter space, as we now discuss.

\section{Freeze-in}

In the freeze-in process, $\theta$ is produced by the annihilation or decay of a heavier particle $X$, with rates small enough not to thermalize, leading to a less-than-thermal DM population, which reaches a plateau at $T \sim m_{X}$ because, at smaller $T$, the number of $X$ particles is Boltzmann suppressed. If the $X$ particles are the SM particles, as is the case through the Higgs portal, their number densities are known as a function of the temperature (simply given by their thermal distribution down to a temperature well below their mass). As a consequence, the created relic density depends only on the magnitude of the portal.

Let us first consider a value of the coupling $g$ sufficiently small to neglect $\theta$ production at the seesaw scale. (We will see that this is the case for $g \leqq 10^{-3}-10^{-4}$, depending on $m_{\theta}$.) In this case, only the Higgs-portal interactions can account for the observed relic density. As we said above, this mechanism is extremely predictive since, given the conformal relation $m_{\theta}^{2}=\lambda v^{2}$, the rates and hence the relic density depend only on the parameter $m_{\theta}$.

Numerically integrating Eq. (15), the observed relic density in Eq. (20) is obtained for

$$
m_{\theta}=2.76-2.86 \mathrm{MeV},
$$

where we took $m_{h}=120 \mathrm{GeV}$ for definiteness. This result corresponds to the right vertical branch of the thick curve in Fig. 1.

An important difference between freeze-in and freezeout is that, while the latter is generally dominated by the off-shell annihilation, the former is naturally dominated by the on-shell annihilation, i.e., by the decay of the mediator 
involved in the annihilation. For a freeze-out, unless the mediator has a mass $\sim 2 m_{\mathrm{DM}}$, the decay has a small effect because, when the off-shell annihilation goes out of equilibrium, it has already decoupled (being more Boltzmann suppressed). For a freeze-in instead, the DM production occurs mostly at $T$ of order the mediator mass, or slightly below, where the Boltzmann suppression is still mild. As a result, the decay, which involves fewer (small) couplings, is naturally dominant, $\gamma_{D_{h}}>\gamma_{\text {annih. }}$. This is shown in Fig. 2 where the various reaction densities are plotted for $m_{h}=$ $120 \mathrm{GeV}$ and $m_{\theta}=2.8 \mathrm{MeV}$. In the approximation where only the decay is included, the Boltzmann equation can be integrated analytically, giving [8]

$$
Y_{\theta} \simeq N_{\theta} \frac{135 g_{h}}{8 \pi^{3}(1.66) g_{*}^{s} \sqrt{g_{*}^{\rho}}} \frac{M_{P} \Gamma(h \rightarrow \theta \theta)}{m_{h}^{2}},
$$

where $g_{h}=1$. This analytic result turns out to differ from the numerical result by less than $1 \%$.

To understand Eq. (22), let us first discuss why the freeze-in is infrared dominated, with most of the $\theta$ production occurring at $T \lesssim m_{h}$ (just before $n_{h}^{\text {eq }}$ gets too Boltzmann suppressed). The $\theta$ number density produced at temperature $T$ is essentially given by the number of decays per unit time occurring at this temperature, $n_{h}^{\mathrm{eq}}\left(m_{h} / T\right) \Gamma(h \rightarrow \theta \theta)$, times the number of $\theta$ particles produced per decay, $N_{\theta}$, times the Hubble time, $1 / H$. Therefore, properly taking into account thermal averaging, one has $Y_{\theta} \simeq \gamma_{D h} /(H s)$, which is nothing but the rate of thermalization $\gamma_{D h} /\left(n_{\theta}^{\mathrm{eq}} H\right)$ shown in Fig. 2, times the relativistic value of $Y_{\theta}^{\mathrm{eq}}$. Thus, one obtains $Y_{\theta} \propto$ $M_{P} m_{h} \Gamma(h \rightarrow \theta \theta) / T^{3}$, which increases as $T$ decreases.

Hence, one finds $Y_{\theta}=c\left[N_{\theta} n_{h}^{\mathrm{eq}} \Gamma(h \rightarrow \theta \theta) /(H s)\right]_{T=m_{h}}$, where $c$ is a numerical factor which turns out to be equal to $(3 \pi / 2) / K_{2}(1) \simeq 2$.9. It is larger than 1 because, as Fig. 2 shows, the maximum value of the decay reaction density occurs at $T \sim m_{h} / 3.5$, rather than at $T \sim m_{h}$ [15]. With this in mind, and taking into account the dependence $\Gamma(h \rightarrow \theta \theta) \propto \lambda^{2} / m_{h} \propto m_{\theta}^{4} / m_{h}$, the DM relic density from the Higgs decay scales as $m_{\theta}^{5} / m_{h}^{3}$, so that the value of $m_{\theta}$ needed varies very little with the exact value of the relic density, and the larger the Higgs mass is, the larger $m_{\theta}$ has to be. For example, for $m_{h}=$ $140,180,300 \mathrm{GeV}$, one gets the observed relic density for $m_{\theta}=3.0,3.6,4.9 \mathrm{MeV}$ instead of the $2.8 \mathrm{MeV}$ obtained in Eq. (21).

It is interesting to discuss the contribution of each scattering channel separately, even though they have a small effect with respect to the decay. As can be seen from Fig. 2, among the scattering channels, the $W$ channel (red curve) gives a contribution slightly larger than the $Z$ (blue curve) and $h$ (green curve) contributions, and much larger than the $b$ (purple curve) and $t$ (orange curve) ones. Numerically, for $m_{h}=120 \mathrm{GeV}$, the $W, Z, h, b$, and $t$ scatterings contribute to the total number of $\theta$ particles produced in the proportions $1: 0.4: 0.4: 4 \times 10^{-3}: 10^{-1}$, respectively, while the $W$ scattering alone gives a contribution 500 times smaller than the decay channel.

Similar to the decay, at a temperature $T \gtrsim m_{a}$, a scattering process $a a \rightarrow \theta \theta$ gives a number density $Y_{\theta}^{a} \simeq$ $\gamma_{\text {annih }}^{a} /(H s)$, which is infrared dominated, too. Again, this is nothing but the thermalization rate $\gamma_{\mathrm{annih}}^{a} /\left(n_{\theta}^{\mathrm{eq}} H\right)$, displayed in Fig. 2, up to the multiplicative constant $Y_{\theta}^{\mathrm{eq}}$. One gets $Y_{\theta}^{a}=c_{a}\left[\gamma_{\text {annih }}^{a} /(H s)\right]_{T=m_{a}}$, where $c_{a}$ is a numerical factor which, unlike for the decay, does not take a unique value. It depends on $m_{\theta}$ as well as on the $s$ dependence of the scattering cross section (which fixes the position of the peak of the reaction densities in Fig. 2). For the $W, Z$ and $h$ channels, $c_{a} \simeq 2.2$, whereas for the fermion channels, $c_{t} \simeq$ 1.7 and $c_{b} \simeq 6$. One understands consequently why the $W$, $Z$, and $h$ channels, which give contributions $Y_{\theta} \propto$ $M_{P} / m_{W, Z, h}$, dominate over the $b$ channel, which gives $Y_{\theta} \propto m_{b}^{3} M_{P} / m_{h}^{4}$. As for the top channel, it is suppressed, too, because, for $s \gtrsim 4 m_{t}^{2}$, it is more threshold suppressed than the other channels [see Eqs. (9)-(12)], and, for higher $s$, it gets quickly suppressed by the $m_{t}^{2} / s^{2}$ asymptotic behavior of the top cross section (as compared to the $1 / \mathrm{s}$ asymptotic behavior of the $W, Z$, and $h$ cross sections).

Let us now move to the region $m_{\theta}<3 \mathrm{MeV}$, where the Higgs portal alone would lead to a too-small relic density. In this case, the $N$ annihilation process can do the job, as long as the reheating temperature is large enough to produce a thermal population of sterile neutrinos, which we assume [16]. The annihilation rate depends on two free parameters only, $g$ and $m_{\theta}$, with the sterile-neutrino mass $m_{N}$ determined by Eq. (4). From the freeze-in of the reaction $N N \rightarrow \theta \theta$, one obtains as usual $Y_{\theta} \simeq$ $\left[\gamma_{\text {annih }} /(H s)\right]_{T=m_{N}}$. One then finds that the DM relic density scales as $g^{6} m_{\nu} / m_{\theta}$, as confirmed by the numerical integration of the Boltzmann equation.

Our numerical result in the $m_{\theta^{-}} g$ plane is shown in Fig. 1 for $m_{\nu}=0.05 \mathrm{eV}$. The diagonal branch of the black thick line corresponds to a freeze-in through the sterile-neutrino portal, with the correct value of the DM relic density. The required value of the $\theta-N$ coupling is given approximately by $g \simeq 2 \cdot 10^{-3}\left(m_{\theta} / \mathrm{MeV}\right)^{1 / 6}\left(\mathrm{eV} / m_{\nu}\right)^{1 / 6}$. Above (below) the line, the relic density is too large (small). Since the relic density is proportional to the sixth power of $g$, the predicted value of $g$ depends very weakly on the cutoff $\Lambda$, which we fixed in all ournumerical analyses, $k=\log \left(\Lambda^{2} / m_{N}^{2}\right) /\left(8 \pi^{2}\right)=1$.

\section{Nonthermal production}

In general, one may think of nonthermal, more modeldependent, $\theta$ production mechanisms. A class of mechanisms is related to the phase transition at the scale of SSB $f$. At this epoch, (part of) the energy stored in the scalarpotential false vacuum may be transferred to the pNGBs. There could also be cosmic strings produced at the phase transition, which would decay into pNGBs, but their exact density depends on model details. Here, we assume that 
these contributions to $\Omega_{\theta}$ are negligible. This is the case, in particular, if the Universe underwent an inflationary phase at temperatures smaller than $f$. (Note that the reheating temperature can still be larger than $m_{N}=$ $g f / \sqrt{2}$, since the coupling $g$ is much smaller than 1 in our scenario.)

A potential source of nonthermal production of $\mathrm{pNGB}$ dark matter could be provided by the oscillations of the field $\theta$ around the minimum of its effective potential, in case the value of $\theta$ at high temperature is displaced from such minimum, a mechanism that has been well studied in the case of the axion. Let us summarize the axion case: The axion mass is very suppressed above $\Lambda_{\mathrm{QCD}}$; therefore, at high temperatures, the axion behaves as an exact NGB. In this case, at the end of the associated Peccei-Quinn phase transition at scale $f_{\mathrm{PQ}}$, the value of the axion field $a$ can lie in any of the equivalent vacua described by $0 \leq a / f_{\mathrm{PQ}}<$ $2 \pi$. Later, when $m_{a}(T)$ becomes important with respect to the Hubble parameter $H(T)$, the field $a$ begins to oscillate around zero, producing a coherent state of particles at rest with an associated $\Omega_{a}^{\text {oscill }}$. (For a review, see Ref. [18].) One may naïvely think that this picture also applies to our scenario, with a negligible $m_{\theta}(T)$ at high temperature (where the EW symmetry is restored), and the oscillations beginning only at $T \sim \mathrm{TeV}$ when $m_{\theta}$ is generated. If this were the case, taking the values of $f$ and $m_{\theta}$ in the range we considered for the freeze-in production, one would conclude that $\Omega_{\theta}^{\text {oscill }}$ overcloses the Universe, unless the initial value of $\theta$ is tuned to be much smaller than $f$, or unless inflation takes place at temperatures below the $\mathrm{TeV}$ scale.

However, the temperature dependence of $m_{\theta}(T)$ is very different from the temperature dependence of the axion, whose mass is generated nonperturbatively by an anomaly at $\Lambda_{\mathrm{QCD}}$. In our case, $m_{\theta}$ is generated, instead, by an explicit breaking of $U(1)_{X}$, and therefore it does not vanish at high temperatures. On the contrary, we expect $m_{\theta}(T)$ to receive large thermal corrections. Even if the EW symmetry is restored for $T \gtrsim \mathrm{TeV}$, there are contributions to $m_{\theta}$ that are not proportional to the Higgs VEV $v$, such as $\delta m_{\theta}^{2} \sim g^{2} y^{2} \Lambda^{2} /\left(16 \pi^{2}\right)^{2}$. (See the discussion at the end of Sec. IV.) These contributions lead to $m_{\theta}^{2}(T) \simeq$ constant $\cdot T^{2}$ at high temperature. A quantitative estimate of a possible nonthermal production of $\theta$ would then require a nontrivial study of the thermal evolution of the field $\theta$ during and after the phase transition at scale $f$. Still, we just notice that $m_{\theta}(T)$ is typically already larger than $H(T)$ at $T \sim f$. Thus, we argue that the field $\theta$ does not acquire a random initial value of order $f$, but rather it sits in the minimum of the potential already at high temperature, or, in other words, coherent oscillations are strongly suppressed. On the basis of this argument, we assume that $\Omega_{\theta}^{\text {oscill }}$ is negligible and that the thermal freeze-in dominates the DM relic density.

\section{E. Summary}

Our results on the $\theta$ relic density are summarized in Fig. 1, where we show the line $\Omega_{\theta}=\Omega_{\mathrm{DM}}$ in the $m_{\theta^{-}} g$ plane. The behavior of the line with the correct relic density can be easily understood. For $m_{\theta} \simeq 0.15 \mathrm{keV}, g$ must be larger than (or equal to) the value needed to thermalize $\theta$. For progressively larger $m_{\theta}$, the value of $g$ should correspond to less and less thermalization. For $0.15 \mathrm{keV}<m_{\theta}<3 \mathrm{MeV}$, one can see that the required coupling $g$ is progressively smaller than the thermalization value, indicated by the thin diagonal line in Fig. 1. When one approaches $m_{\theta} \simeq 3 \mathrm{MeV}, g$ becomes rapidly smaller as the Higgs portal begins to contribute to the $\theta$ number density. Once the Higgs portal produces by itself the correct amount of $\theta$ particles, $g$ must be small enough to make the sterile-neutrino freeze-in negligible.

We conclude that the observed DM relic density can be obtained thermally for

$$
0.15 \mathrm{keV} \lesssim m_{\theta} \lesssim 3 \mathrm{MeV},
$$

where the exact upper value depends on the Higgs mass, as discussed in Sec. III C. Note that this upper bound holds as an absolute prediction as soon as the Higgs portal dominates the DM production. In particular, this is necessarily the case if the reheating temperature lies below the sterileneutrino-mass scale. More generally, the prediction $m_{\theta} \simeq$ $3 \mathrm{MeV}$ holds for any pNGB whose Higgs-portal interaction $\lambda$ gives the dominant contribution to its mass and relic density, independent of the associated global symmetry and of the source of explicit symmetry breaking which induces $\lambda$. In particular, the SSB scale may be different from the seesaw scale.

Of course the prediction for $m_{\theta}$ would change if the number of DM particles was not negligible already at temperatures higher than the EW scale. In this case, one has to produce less of them through freeze-in, which means that $m_{\theta}$ has to be smaller. In other words, $m_{\theta} \simeq 3 \mathrm{MeV}$ constitutes an absolute upper bound for the freeze-in mechanism, and it holds as soon as the freeze-in production dominates over the initial population.

\section{APPROXIMATE SYMMETRIES OF THE SEESAW SECTOR}

In this section, we consider the SM augmented with sterile neutrinos $\nu_{i}^{c}$, with a global symmetry broken spontaneously at the seesaw scale, and we discuss in some detail the generation of a mass for the associated pNGB.

Let us consider the most general Yukawa interactions to be added to the SM in the presence of gauge-singlet fermions,

$$
-\mathcal{L}_{\nu^{c}}=l_{\alpha} m_{\alpha j} \nu_{j}^{c}\left(\frac{H}{v}\right)+\frac{1}{2} \nu_{i}^{c} M_{i j} \nu_{j}^{c}+\text { H.c., }
$$

where $l_{\alpha}$ are the lepton doublets and $H$ is the Higgs doublet, whose neutral component acquires a VEV of 
$v=174 \mathrm{GeV}$. Here and in the following analysis, the mass parameters $m_{\alpha j}$ and $M_{i j}$ are intended in a generalized sense as dynamical scalar fields that may or may not acquire a nonzero VEV. Thus, the above Lagrangian has a global $U(1)_{L}$ symmetry with the following leptonnumber assignments:

$$
\begin{gathered}
L(l)=+1, \quad L\left(\nu^{c}\right)=-1, \\
L(H)=L(m)=0, \quad L(M)=+2,
\end{gathered}
$$

where we dropped flavor indices. When $M$ acquires a VEV, $U(1)_{L}$ is spontaneously broken, and a massless NGB appears in the spectrum of the theory: the singlet Majoron [1]. One can write more explicitly

$$
M_{i j}=g_{i j} \Phi, \quad \Phi \equiv \frac{\rho}{\sqrt{2}} e^{i \theta / f},
$$

where $\Phi$ is a complex scalar with $L=2$, the $\operatorname{VEV}\langle\rho\rangle=f$ spontaneously breaks the lepton number, and $\theta$ is the Majoron.

Various possible sources of $U(1)_{L}$-explicit breaking come from other sectors of the Lagrangian. There may be soft terms in the scalar potential involving $\Phi$ which break the lepton number; their mass scale, whose size is arbitrary, determines the induced Majoron mass (see, e.g., [7]). Another possible source of $U(1)_{L}$-explicit breaking is quantum gravity effects at the Planck scale $M_{\mathrm{P}}$, which can, in general, break all nongauge symmetries. Assuming that these effects are suppressed by powers of $M_{\mathrm{P}}$, they can be used to generate a Majoron mass at the $\mathrm{keV}$ scale [2,3], which has been extensively studied as a DM candidate [3-6].

In this paper, we consider a different source of explicit breaking of global symmetries, provided by the set of the Yukawa couplings [9]. This source allows us to relate the size of the pNGB parameters to the fermion mass scales already present in the theory. We will focus on symmetries of the Lagrangian in Eq. (24) other than $U(1)_{L}$, with each lepton carrying, in general, a different charge. In this case, the symmetries are respected only by some matrix elements $m_{\alpha j}$ and $M_{i j}$, and they are explicitly broken if some other matrix elements are nonzero. The mass and couplings of the pNGBs are completely determined by the seesaw parameters and by the choice of the cutoff, since they can receive cutoff-dependent quantum corrections.

The pNGB mass is, in general, quadratically sensitive to the cutoff. In order to understand the origin of quadratic divergences and the mechanism to remove them, consider first the explicit breaking of a $U(1)$ symmetry in a theory with only one sterile neutrino, $\nu^{c}$ :

$$
\begin{aligned}
-\mathcal{L}_{\nu^{c}}= & \frac{1}{2} \nu^{c}\left(M_{a} e^{i \theta / f}+M_{b}\right) \nu^{c}+\text { H.c. } \\
= & \frac{1}{2}\left(M_{a}+M_{b}\right) \bar{N} N-\frac{i M_{a}}{2 f} \theta \bar{N} \gamma_{5} N \\
& -\frac{M_{a}}{4 f^{2}} \theta^{2} \bar{N} N+\mathcal{O}\left(\theta^{3} / f^{3}\right),
\end{aligned}
$$

where $M_{a, b}$ are real mass parameters. Here, $M_{a}$ is generated by the spontaneous breaking of the $U(1)$ symmetry associated with the NGB $\theta$, while $M_{b}$ breaks this symmetry explicitly. It is instructive to compute the fermion loops generating the pNGB mass term, $m_{\theta}^{2} \theta^{2} / 2$, using the righthand side of Eq. (27): If $M_{b}$ is zero, the two relevant oneloop diagrams cancel each other, as expected for an exact NGB. However, in the presence of the explicit breaking, a nonzero, quadratically divergent contribution is left, which is given by

$$
m_{\theta}^{2} \sim \frac{1}{8 \pi^{2}} \frac{M_{a} M_{b}}{f^{2}} \Lambda^{2} .
$$

The effective theory below the scale $f$ may still contain a light scalar $\theta$, since $m_{\theta}$ can be parametrically small, but its NGB nature is obscured by the quadratic dependence on the details of the ultraviolet completion.

In the presence of more than one fermion family, one can define several family-dependent $U(1)$ symmetries. In general, they are explicitly broken by some mass-matrix entries. However, certain $U(1)$ 's will be broken only when several entries are nonzero at the same time: This is the key to reducing the degree of divergence of the radiative contribution to $m_{\theta}$, where $\theta$ 's are the associated pNGBs [9]. This fact can be understood in the language of the effective potential $V_{\text {eff }}$, considering the full (active + sterile) neutrino-mass matrix $\mathcal{M}$. The term in the $V_{\text {eff }}$ quadratic in the mass matrix, $\sim \operatorname{Tr}\left(\mathcal{M} \mathcal{M}^{\dagger}\right) \Lambda^{2}$, is invariant under certain $U(1)$ symmetries, that is, it does not depend on the associated pNGBs. Thus, the potential of these pNGBs contains at most terms quartic in the mass matrix, $\sim \operatorname{Tr}\left(\mathcal{M} \mathcal{M}^{\dagger} \mathcal{M} \mathcal{M}^{\dagger}\right) \log \Lambda^{2}$, which are only logarithmically sensitive to the cutoff. (For an application of this idea to $\mathrm{eV}$-scale sterile neutrinos, with the pNGB playing the role of dark energy, see [19].) Of course, non-Abelian symmetries are also possible, with several NGBs and, potentially, qualitatively different phenomena, but this extension is not needed for our purposes and is not considered in this paper.

To identify the combination of matrix entries that induces a pNGB mass, we rewrite Eq. (24) with the replacement $l_{\alpha}(H / v) \rightarrow \nu_{\alpha}$, in the minimal case of two sterile neutrinos, which are sufficient for realistic light-neutrino masses:

$$
\begin{aligned}
-\mathcal{L}_{\nu^{c}}= & \nu_{\alpha}\left(m_{\alpha 1} m_{\alpha 2}\right)\left(\begin{array}{l}
\nu_{1}^{c} \\
\nu_{2}^{c}
\end{array}\right) \\
& +\frac{1}{2}\left(\nu_{1}^{c} \nu_{2}^{c}\right)\left(\begin{array}{ll}
M_{11} & M_{12} \\
M_{12} & M_{22}
\end{array}\right)\left(\begin{array}{l}
\nu_{1}^{c} \\
\nu_{2}^{c}
\end{array}\right)+\text { H.c. }
\end{aligned}
$$

Here, a sum over $n_{f}=3$ active flavors is understood $(\alpha=e, \mu, \tau)$. Let us identify the matrix entries whose phases can be removed. In general, $2 n_{f}+3$ mass terms have a phase. One can absorb $n_{f}+2$ of these phases by redefining the $n_{f}+2$ neutrino fields present in Eq. (29). As a 
result, there remain $n_{f}+1$ complex matrix entries in Eq. (29). (There are either $n_{f}$ phases in $m$ and one in $M$, or, equivalently, $n_{f}-1$ phases in $m$ and two in $M$.) When one sets these $n_{f}+1$ entries to zero, there are no physical phases left.

Now, suppose these $n_{f}+1$ complex entries vanish, and the nonzero entries are generated by a set of scalar fields, $\Phi_{a} \equiv \rho_{a} \times \exp \left(i \theta_{a} / f_{a}\right) / \sqrt{2}$, acquiring a $\operatorname{VEV}$ of $\left\langle\rho_{a}\right\rangle=$ $f_{a}$. Then, by an appropriate redefinition of the lepton fields, one can remove all the phase fields $\theta_{a}$ from the Yukawa Lagrangian of Eq. (29). Thus, these fields will have only derivative couplings to the leptons, resulting from the redefined lepton kinetic terms. This means that the Lagrangian has exact $U(1)$ symmetries, broken spontaneously by $\langle\Phi\rangle$ 's, and that $\theta_{a}$ are exact NGBs.

When one of the zero-matrix entries is switched on, however, it is no longer possible to remove all the phases. This fact means that one $U(1)$ is explicitly broken, with the associated pNGB $\theta$ acquiring nonderivative couplings. Thus, a nonzero $m_{\theta}$ is generated by neutrino one-loop diagrams. To estimate $m_{\theta}$, consider the set of the $\left(n_{f}+\right.$ 3 ) nonzero-matrix entries. One can check that $\theta$ could be rotated away from Eq. (29) if any out of four of these entries was switched off. This means that a $U(1)$ symmetry is recovered when any entry is put to zero, and, therefore, $m_{\theta}$ must be proportional to the product of the four entries. As a consequence, quadratically divergent contributions to $m_{\theta}$ are absent.

The $\theta$ mass can be controlled by the entries of $M$ only, by those of $m$ only, or by both. Let us specify three models with a $U(1)_{X}$ symmetry, which are representative of these three generic possibilities:

(i) The symmetry is broken explicitly in the singletneutrino sector. Two independent entries of the Majorana mass matrix $M$ are allowed by $U(1)_{X}$, while the third is forbidden. For example, take $X\left(\nu_{1}^{c}\right)=0, X\left(\nu_{2}^{c}\right)=1$, and one scalar field $\Phi$ with $X(\Phi)=-2$. Then, $M_{11}$ is allowed and $M_{22}$ is generated by the VEV of $\Phi$, while $M_{12}=0$. The NGB coupling to neutrinos reads $M_{22} \exp (i \theta / f) \nu_{2}^{c} \nu_{2}^{c} / 2$. (The Dirac neutrino sector is not relevant here: The $m$ entries may or may not respect $U(1)_{X}$. In any case, the leading symmetry-breaking effects are controlled by $M$.) When $M_{12}$ is different from zero, neutrino loops give a nonzero contribution to $m_{\theta}$ as long as they contain four appropriate mass insertions:

$$
m_{\theta}^{2} \sim \frac{1}{8 \pi^{2}} \frac{M_{11} M_{12} M_{22} M_{12}}{f^{2}} \log \frac{\Lambda^{2}}{\mu^{2}},
$$

where we include a loop-suppression factor and $\mu$ is the renormalization scale, which can be taken of the order of the sterile-neutrino masses. The cancellation of quadratic divergences as well as the structure of the nonvanishing contribution to $m_{\theta}$ are dictated by our symmetry argument, and they can both be explicitly checked by computing the loops. (ii) The symmetry is broken explicitly only within the Dirac neutrino sector. The entries on a given row of the Dirac mass matrix $m$ are both allowed, while, in the other rows, only one entry is allowed. For example, take $X\left(\nu_{e}\right)=1, X\left(\nu_{\mu, \tau}\right)=3, X\left(\nu_{1}^{c}\right)=-1$, $X\left(\nu_{2}^{c}\right)=1$, and a scalar field $\phi$ with $X(\phi)=-2$. Then, $m_{e 1}$ is allowed, $m_{e 2}, m_{\mu 1}$ and $m_{\tau 1}$ are generated by the VEV of $\phi$, and $m_{\mu 2}=m_{\tau 2}=0$. (For simplicity, we suppose that the scalar fields $\phi$ and $\Phi$ that can contribute to $m$ and to $M$ belong to different sets. The singlet-neutrino sector is assumed to respect $U(1)_{X}$ : In the present example, $M_{12}$ is allowed, thus giving an equal mass to the two sterile neutrinos.) This case closely reproduces the scenario discussed by Hill and Ross [9], which dealt with the quark Dirac mass matrix. Once the entries explicitly breaking $U(1)_{X}$ are switched on in $m$, one obtains

$$
m_{\theta}^{2} \sim \frac{1}{8 \pi^{2}} \frac{m_{\alpha 1} m_{\alpha 2} m_{\beta 2} m_{\beta 1}}{f^{2}} \log \frac{\Lambda^{2}}{\mu^{2}}, \quad \alpha \neq \beta .
$$

(iii) The symmetry is broken explicitly only by the interplay of the Dirac and Majorana neutrino-mass matrices. One possibility is that $U(1)_{X}$ allows for all the entries of $M$, e.g., taking the charges $X\left(\nu_{1}^{c}\right)=$ $-1, X\left(\nu_{2}^{c}\right)=1$, and $X(\Phi)=2$; this symmetry can allow only one entry between $m_{\alpha 1}$ and $m_{\alpha 2}$, depending on the charge of $\nu_{\alpha}$. Another possibility is that $U(1)_{X}$ allows for all the entries of $m$ but forbids two entries of $M$, e.g., taking the charges $X\left(\nu_{\alpha}\right)=$ $X\left(\nu_{1}^{c}\right)=0$ and $X\left(\nu_{2}^{c}\right)=-X(\phi) \neq 0$. As usual, the mass of the NGB $\theta$ is generated when one zero entry is switched on. One finds

$$
m_{\theta}^{2} \sim \frac{1}{8 \pi^{2}} \frac{M_{i j} M_{k l} m_{\alpha 1} m_{\alpha 2}}{f^{2}} \log \frac{\Lambda^{2}}{\mu^{2}}, \quad i j \neq k l .
$$

To roughly quantify the energy scales under discussion, let us indicate with $M=g f(m=y v)$ a generic seesaw (electroweak) scale, which is possibly suppressed by a small Yukawa coupling $g(y)$ with respect to the $U(1)_{X}$ SSB scale $f$ (the EW scale $v$ ). Let us also introduce the neutrino-mass scale $m_{\nu}=m^{2} / M$. Up to a loop suppression, in the three cases, $m_{\theta}$ is of order (i) $(M / f) M=g M$, at or below the seesaw scale; (ii) $(m / f) m=g m_{\nu}$, at or below the neutrino-mass scale; (iii) $(M / f) m=g m$, at or below the electroweak scale. In the following discussion, we will concentrate on the last possibility, since we are interested in scalar DM candidates below the electroweak scale.

Before we move to the other properties of the pNGB relevant for DM, some comments are in order to assess the soundness of the above estimate for the pNGB mass $m_{\theta}$. We have seen that, in general, in the presence of explicit breaking of the associated global symmetry, $m_{\theta}$ can be sensitive quadratically to the cutoff of the theory. However, 
if the global symmetry is broken only by the contemporary presence of several couplings, then $m_{\theta}$ is proportional to the product of all these couplings. In this case, the Feynman diagrams contributing to the pNGB mass will have a lower degree of divergence, and thus quadratic divergences vanish. This possibility, often employed in model building in the past, has recently been extensively used in the context of little Higgs models, under the name of "collective breaking." (See Ref. [10] for reviews.) The motivation is to stabilize the electroweak scale against large quantum corrections. In these models, the Higgs is a NGB of a symmetry broken spontaneously at the scale $f_{\text {ew }} \sim \mathrm{TeV}$, whose mass is generated at one-loop level by two couplings $y_{1,2}$, whose contemporary presence explicitly breaks the symmetry. As a consequence, $m_{h}^{2} \sim$ $y_{1}^{2} y_{2}^{2} f_{\text {ew }}^{2} \log \left(\Lambda^{2} / \mu^{2}\right) /\left(16 \pi^{2}\right)$, that is, the sensitivity to the cutoff $\Lambda$ is only logarithmic. However, in general, the quadratic divergence reappears at the two-loop level, with a correction to the Higgs mass $\delta m_{h}^{2} \sim y_{1}^{2} y_{2}^{2} \Lambda^{2} /$ $\left(16 \pi^{2}\right)^{2}$, which indicates that $\Lambda$ cannot be larger than approximately $4 \pi f_{\text {ew }}$, in order for the theory to remain natural.

It is instructive to compare this little Higgs scenario with our scenario, where the DM candidate $\theta$ is a pNGB associated with SSB at the scale $f$, of the order of the seesaw scale. In the latter case, the pNGB mass generated at one-loop can be written schematically as $m_{\theta}^{2} \sim$ $g^{2} y^{2} v^{2} \log \left(\Lambda^{2} / \mu^{2}\right) /\left(16 \pi^{2}\right)$. The mass $m_{\theta}$ is proportional not to $f$, but rather to the electroweak scale $v$. Besides, it can be much smaller than $v$ because of the four powers of Yukawa couplings and the loop suppression. This is true even with a huge cutoff $\Lambda$, since the dependence on it is only logarithmic. However, at higher orders, the quadratic divergence may reappear, e.g., through a two-loop diagram with a virtual Higgs exchanged across the neutrino loop, leading to $m_{\theta}^{2} \sim g^{2} y^{2} \Lambda^{2} /\left(16 \pi^{2}\right)^{2}$. Although this result is not surprising, since the mechanism we adopted explains why the pNGB mass is related to the EW scale, it does not address the stability of the EW scale against radiative corrections. In other words, as already remarked by Hill and Ross [9], the Higgs sensitivity to quadratic corrections also enters in $m_{\theta}$ at higher order. In order for this two-loop correction to be negligible with respect to the one-loop estimate, one needs the cutoff of the Higgs boson loops, $\Lambda_{H}$, to be smaller than approximately $4 \pi v$. (By enlarging the global symmetry, it may also be possible to remove the two-loop quadratic divergence; see, e.g., [19], but, in general, higher orders reintroduce the problem.) If one wants to stabilize a theory which includes a scale much larger than $v$ (e.g., the scale $f$ ), one must address the usual hierarchy problem, e.g., postulating supersymmetry broken at $\Lambda_{\text {susy }} \sim \mathrm{TeV}$, or a strongly interacting sector that condenses at $\Lambda_{c} \sim \mathrm{TeV}$ dynamically generating the EW scale. In this paper, we assume that the stability of the EW scale is realized, and thus we can adopt our one-loop estimates for the pNGB mass and couplings in order to study the phenomenology.

\section{A. A seesaw model leading to the Higgs portal}

Let us consider in some detail a specific $U(1)_{X}$ symmetry of the neutrino sector, such that the pNGB $\theta$ radiatively acquires a coupling to the SM Higgs. Take the sterileneutrino charges $X\left(\nu_{1}^{c}\right)=-1$ and $X\left(\nu_{2}^{c}\right)=1$, and a scalar field $\Phi$ with charge $X(\Phi)=2$, whose VEV breaks the symmetry spontaneously. The interaction Lagrangian involving the sterile neutrinos and the pNGB $\theta$ reads

$$
\begin{aligned}
-\mathcal{L}_{\nu^{c}-\theta}= & l_{\alpha}\left(m_{\alpha 1} m_{\alpha 2}\right) \frac{H}{v}\left(\begin{array}{c}
\nu_{1}^{c} \\
\nu_{2}^{c}
\end{array}\right) \\
& +\frac{1}{2}\left(\nu_{1}^{c} \nu_{2}^{c}\right)\left(\begin{array}{cc}
M_{11} e^{i \theta / f} & M_{12} \\
M_{12} & M_{22} e^{-i \theta / f}
\end{array}\right)\left(\begin{array}{c}
\nu_{1}^{c} \\
\nu_{2}^{c}
\end{array}\right) \\
& + \text { H.c. }
\end{aligned}
$$

Here, $M_{12}$ is a mass term allowed by $U(1)_{X}$, while $M_{11}$ and $M_{22}$ are generated after SSB. The symmetry is broken explicitly by either $m_{\alpha 1}$ or $m_{\alpha 2}$, depending on the $U(1)_{X}$ charge assigned to the lepton doublet $l_{\alpha}$ [20]. Note that $\theta$ can be rotated away by rephasing the neutrino fields if $m_{\alpha 1}=0$ or $m_{\alpha 2}=0$. In this case, the symmetry is restored and $\theta$ is a true NGB. The dependence on $\theta$ can also be removed when two independent entries of $M$ are vanishing; therefore, the symmetry-breaking effects must also vanish in this limit. As we discussed in the previous section, this need for four different couplings to break the symmetry is the key to canceling quadratic divergences [21].

The interactions in Eq. (33) generate an effective potential for the pNGB $\theta$. We assumed that $M_{i j}$ and $m_{\alpha i}$ are real and positive (see discussion in the next paragraph), and we performed the one-loop computation of the effective potential, which can be written as

$$
V_{\text {eff }}=\frac{\lambda}{2} \theta \theta H^{\dagger} H+\mathcal{O}\left(\theta^{4}\right) .
$$

We find that the quadratically divergent contributions cancel explicitly, as expected, and the logarithmically divergent contributions give

$$
\lambda \simeq \frac{1}{4 \pi^{2}} \frac{M_{12}\left(M_{11}+M_{22}\right)}{f^{2}} \frac{\sum_{\alpha} m_{\alpha 1} m_{\alpha 2}}{v^{2}} \log \frac{\Lambda^{2}}{\mu^{2}},
$$

with a renormalization scale $\mu \sim M_{i j}$ and up to tiny corrections of higher order in $m_{\alpha i} / M_{k l}$. This type of model is particularly predictive, because the pNGB mass is generated by the same loops that generate $\lambda$; that is, $m_{\theta}$ is obtained from Eq. (34) by replacing the Higgs with its VEV, $m_{\theta}^{2}=\lambda v^{2}$. By taking a common value $m_{N}=g f / \sqrt{2}$ $(m=y v)$ for each entry of the Majorana (Dirac) neutrinomass matrix, Eq. (35) reduces to Eq. (3), up to a factor $2 n_{f}$ that accounts for the sum-over-flavor indices. As we saw in Sec. III, the value of the coupling $\lambda$ has a crucial role for 
the (partial) thermalization of $\theta$ and thus for the determination of its relic density.

A comment on the $C P$ symmetry is in order. In general, the mass terms $M_{i j}$ and $m_{\alpha i}$ may be complex, that is, they may carry phases that cannot be removed by a redefinition of the fields. These phases correspond to an explicit breaking of $C P$. In this case, $\theta$ would have both scalar and pseudoscalar couplings to the fermions, with characteristic phenomenological signatures [9]. Moreover, the effective potential would contain terms odd in $\theta$, such as $\mu \theta H^{\dagger} H$ (with $\mu \ll v$ ), that induce a VEV for $\theta$ and a small mixing with the Higgs. This oddity may endanger the stability of $\theta$, since it would couple linearly to SM particles. Still, these couplings can be very small and, in addition, the $\theta$ decays may be kinematically forbidden if $m_{\theta}$ is sufficiently small. In this paper, we do not investigate this more complicated $C P$-violating possibility; rather, we assume that $C P$ is a good symmetry of the seesaw sector. In this case, $M_{i j}$ and $m_{\alpha i}$ in Eq. (33) are all real, and $\theta$ preserves its pseudoscalar nature, since Eq. (33) is invariant under a $C P$ transformation with $\theta \rightarrow-\theta$. Usually, the DM stability requires an additional (discrete) symmetry that forbids its decay into SM particles. In the present scenario, the $C P$ symmetry itself guarantees that $\theta$ couples linearly only to the heavy sterile neutrinos, and we will see in Sec. V that this circumstance makes $\theta$ sufficiently long-lived.

\section{CONSTRAINTS ON THE PNGB LIFETIME}

We first derive the couplings of $\theta$ to the SM fermions and gauge bosons, and compare the $\theta$ decay width into light SM particles with the lifetime of the Universe. Subsequently, assuming that $\theta$ particles account for the whole DM density, we discuss the more stringent astrophysical and cosmological constraints that exist on the decay of $\theta$ to neutrinos, electrons, and photons.

\section{A. $\theta$ couplings to the SM particles}

The pNGB lifetime is determined by its couplings to light fermions. These couplings come from the $\theta-N$ interaction, through the $\nu-N$ mixing induced by the Dirac neutrino masses. In this way, $\theta$ can decay to light neutrinos (at tree level) and to charged fermions (at one-loop level). In turn, these couplings to SM fermions could induce, through triangle-loop diagrams, couplings to SM gauge bosons. These decays should be sufficiently slow to make the DM lifetime longer than the age of the Universe, $\tau_{0} \simeq$ $5 \times 10^{17} \mathrm{~s}$. This provides interesting constraints on the seesaw parameters, as we now discuss.

Neutrinos.-At tree level, $\theta$ couples only to light neutrinos, as follows:

$$
\begin{aligned}
\mathcal{L}_{\theta \nu \nu} & =\frac{i}{2} \sum_{\alpha \beta} \frac{\left(\mu_{\nu}\right)_{\alpha \beta}}{f} \bar{\nu}_{\alpha} \gamma_{5} \nu_{\beta} \theta \\
\left(\mu_{\nu}\right)_{\alpha \beta} & \equiv-\sum_{i j} X_{i j}\left(m M^{-1}\right)_{\alpha i} M_{i j}\left(M^{-1} m^{T}\right)_{j \beta} .
\end{aligned}
$$

Here $X_{i j}$ is the power of $e^{i \theta / f}$ associated with the sterileneutrino-mass-matrix entry $M_{i j}$. In the singlet Majoron model $X_{i j}=1$ for all $i, j$, therefore, one finds $\mu_{\nu}=m_{\nu} \equiv$ $-m M^{-1} m^{T}$, which is the usual seesaw formula. On the contrary, in our models based on a family-dependent $U(1)_{X}$ symmetry, $\theta$ couples differently to each entry of $M$, as, for example, in the model of Eq. (33). This Lagrangian leads to a total decay width (into both neutrinos and antineutrinos) of

$$
\Gamma(\theta \rightarrow \nu \nu)=\frac{1}{16 \pi} g_{\theta \nu \nu}^{2} m_{\theta}, \quad g_{\theta \nu \nu}^{2} \equiv \frac{\operatorname{Tr}\left(\mu_{\nu}^{\dagger} \mu_{\nu}\right)}{f^{2}},
$$

where we neglected $\left(m_{\nu i} / m_{\theta}\right)^{2}$ corrections in the phase space, $m_{\nu i}$ being the light-neutrino-mass eigenvalues. In the Majoron case, one obtains $g_{\theta \nu \nu}^{2}=\sum_{i} m_{\nu i}^{2} / f^{2}$. This width is smaller than $1 / \tau_{0}$ for $g_{\theta \nu \nu} \lesssim 3 \times$ $10^{-19}\left(\mathrm{MeV} / m_{\theta}\right)^{1 / 2}$. Such tiny coupling is natural for $\theta$ because the pNGB couplings are suppressed by the SSB scale $f$ : One needs $f \gtrsim 3 \times 10^{9} \mathrm{GeV}\left(m_{\nu} / \mathrm{eV}\right)\left(m_{\theta} / \mathrm{MeV}\right)^{1 / 2}$, where we use the one-family approximation, $g_{\theta \nu \nu} \simeq m_{\nu} / f$. To translate this bound in the $m_{\theta^{-}} g$ plane, we insert the relation $f=\sqrt{2} m_{N} / g$ into Eq. (4), which gives

$$
g_{\theta \nu \nu} \simeq 10^{-21}\left(\frac{\mathrm{MeV}}{m_{\theta}}\right)^{2}\left(\frac{g}{10^{-3}}\right)^{3}\left(\frac{m_{\nu}}{\mathrm{eV}}\right)^{2} k .
$$

The condition $1 / \Gamma(\theta \rightarrow \nu \nu)>\tau_{0}$ excludes the region above the blue dashed line in Figs. 3 and 4.

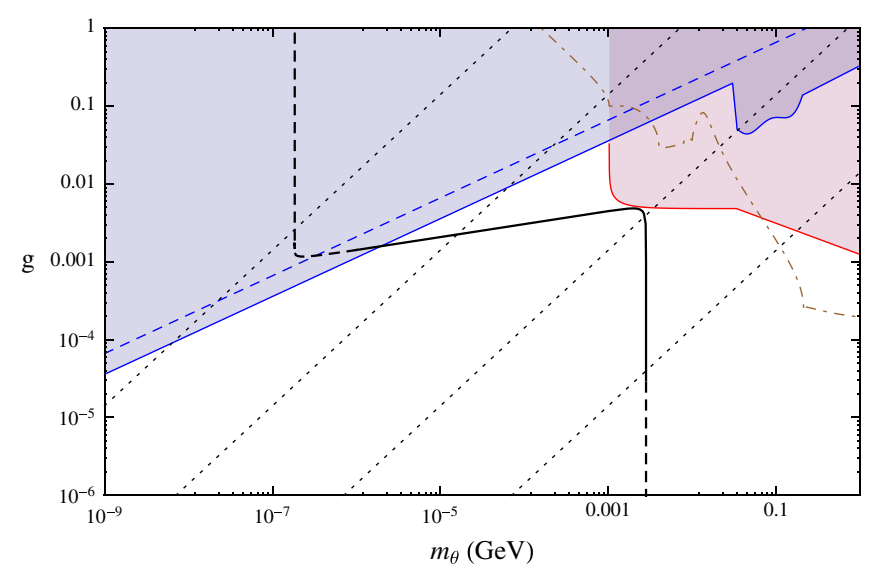

FIG. 3. The constraints on the DM lifetime in the $m_{\theta^{-}} g$ plane, for $m_{\nu}=0.05 \mathrm{eV}$. The thick black curve, corresponding to the correct DM relic density, as well as the dotted vertical lines, corresponding to constant values of $m_{N}$, are the same as presented in Fig. 1. The curve is dashed for $m_{\theta} \lesssim 1 \mathrm{keV}$, because DM is warm in this region (see the text), and for $g \lesssim 10^{-5}$, because of the theoretical bound $f<M_{P}$, shown in Fig. 1. The blue solid (dashed) line is the upper bound on $g$ from DM decays into neutrinos coming from astrophysics and cosmology (from the Universe lifetime); the blue shaded region is therefore excluded. The red solid line is the analog bound for DM decays into $e^{+} e^{-}$; the red shaded region is correspondingly excluded. Finally, the brown dash-dotted line is a conservative estimate of the upper bound on $g$ from DM decays into photons (see the text). 


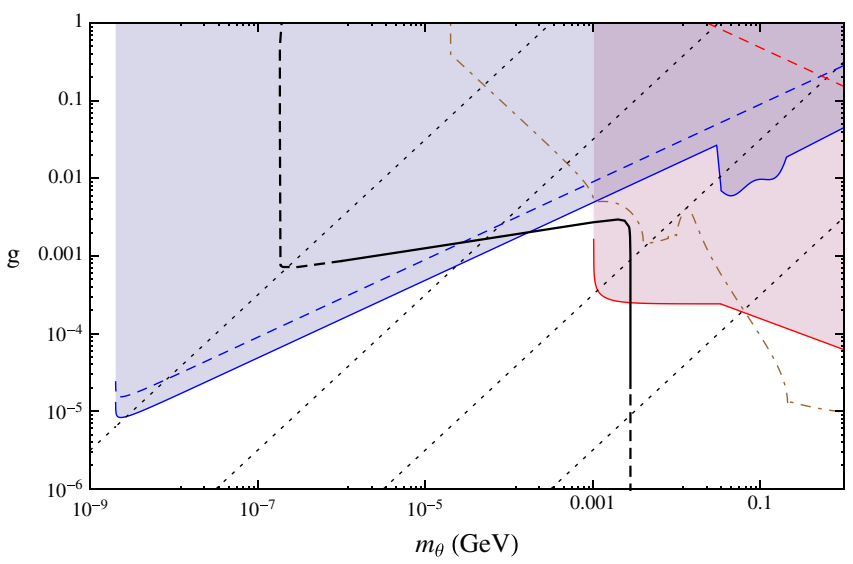

FIG. 4. The same as in Fig. 3, but for a heavier-neutrino-mass scale, $m_{\nu}=1 \mathrm{eV}$. The extra red dashed line is the upper bound from $1 / \Gamma\left(\theta \rightarrow e^{+} e^{-}\right)>\tau_{0}$. (In the case of Fig. 3, the analog line lies entirely in the region $g>1$ and $m_{\theta}>1 \mathrm{GeV}$, which is not displayed.)

Charged fermions.-The pNGB $\theta$ also couples to charged fermions, through EW one-loop diagrams. This effect arises because of the mixing between the sterile and the weakly interacting neutrinos; in particular, it is also operative in the singlet Majoron model [1,22]. The coupling to quarks is induced by a one-loop $\theta-Z$-mixing diagram, with neutrinos in the loop. The coupling to charged leptons is generated by an analog Z-exchange diagram plus a triangle diagram with $W$ exchange. The resulting coupling can be written in a compact form in the one-family approximation, as follows:

$$
\mathcal{L}_{\theta f \bar{f}}=i g_{\theta f \bar{f}} \bar{f} \gamma_{5} f \theta, \quad g_{\theta f \bar{f}}= \pm \frac{G_{F}}{(4 \pi)^{2}} \frac{\sqrt{2} m_{N}}{f} m_{f} m_{\nu},
$$

where the sign is $+(-)$ for up quarks and charged leptons (down quarks), and $G_{F}$ is the Fermi coupling constant. This effective coupling can be suppressed taking only a small $g=\sqrt{2} m_{N} / f$, since all the other parameters in $g_{\theta f \bar{f}}$ can be determined experimentally. The decay width is given by

$$
\Gamma(\theta \rightarrow f \bar{f})=\frac{1}{8 \pi} g_{\theta f f}^{2} m_{\theta}\left(1-\frac{4 m_{f}^{2}}{m_{\theta}^{2}}\right)^{1 / 2} .
$$

We remark that all models where the singlet Majoron is given a mass larger than $1 \mathrm{MeV}$ and plays the role of DM candidate are constrained (or already excluded) by such decays into charged fermions.

In our scenario, the requirement $1 / \Gamma(\theta \rightarrow f \bar{f})>\tau_{0}$ leads to an upper bound $g \leqq 2\left(\mathrm{MeV} / m_{\theta}\right)^{1 / 2}\left(\mathrm{eV} / m_{\nu}\right) \times$ $\left(\mathrm{MeV} / m_{f}\right)$, where we have assumed $m_{\theta} \gg 2 m_{f}$ for simplicity. This bound excludes the region above the red dashed line in Fig. 4. As a consequence, taking into account that $g$ and $m_{\theta}$ are related by Eq. (3), and that the Dirac mass $m=y v$ cannot be larger than about $\mathrm{TeV}$, we conclude that $m_{\theta}$ close to the EW scale would imply a lifetime shorter than $\tau_{0}$. In fact, even stronger constraints on the decay width into charged fermions come from astrophysical and cosmological observations (see Sec. V B), which will lead to a stronger constraint, $m_{\theta}<1 \mathrm{GeV}$. In particular, in this class of models, $\theta$ cannot be the approximately $50 \mathrm{GeV}$ DM candidate produced by the freeze-out of the $\lambda$ interaction with the Higgs, discussed in Sec. III B. One is left with the possibility of a sub-GeV DM candidate, because in this case the decays into charged fermion pairs are sufficiently slow (or forbidden kinematically), and the correct relic density can be generated by the freeze-in mechanism.

In the realistic three-family case, the coupling $g_{\theta f \bar{f}}$ is generically of the same order, but with a complicated dependence on flavor parameters. In particular, one may argue that some cancellation can take place, to reduce the $\theta$ decay width. In addition, we have seen in Sec. IV that the pNGB-mass generation depends crucially on the interplay between the flavor structures of the matrices $m$ and $M$. Therefore, if one were to study the whole parameter space, one should know the explicit dependence of $g_{\theta f \bar{f}}$ on the mass-matrix entries.

For illustration, we display the result for the model in Eq. (33), considering for simplicity only one lepton doublet. Writing the Dirac mass matrix as $m \equiv\left(m_{1} m_{2}\right)$ and keeping terms up to order $\left(m_{i} / M_{j k}\right)^{2}$, the effective coupling of $\theta$ to fermions through the mixing with the $Z$-gauge boson is given by

$$
\begin{aligned}
& \mathcal{L}_{\theta f \bar{f}}^{Z}=i\left(\frac{M_{11}}{f} F_{1}-\frac{M_{22}}{f} F_{2}\right) \frac{2 \sqrt{2} G_{F}}{(4 \pi)^{2}} m_{f} T_{3 f} \bar{f} \gamma_{5} f \theta, \\
& F_{1}=m_{1}^{2}\left(c^{2} s^{2} K+\frac{c^{2}}{M_{1}}+\frac{s^{2}}{M_{2}}\right)-m_{2}^{2} c^{2} s^{2} K \\
&-m_{1} m_{2} c s\left(\left(c^{2}-s^{2}\right) K-\frac{1}{M_{1}}+\frac{1}{M_{2}}\right), \\
& F_{2}= m_{2}^{2}\left(c^{2} s^{2} K+\frac{s^{2}}{M_{1}}+\frac{c^{2}}{M_{2}}\right)-m_{1}^{2} c^{2} s^{2} K \\
&+m_{1} m_{2} c s\left(\left(c^{2}-s^{2}\right) K+\frac{1}{M_{1}}-\frac{1}{M_{2}}\right),
\end{aligned}
$$

where $T_{3 f}$ is the third isospin component of the left-handed part of the fermion $f, M_{1,2}$ are the eigenvalues of the matrix $M$, which is diagonalized by a rotation of angle $\delta$, defined by $\tan 2 \delta=2 M_{12} /\left(M_{11}-M_{22}\right)$, and we denote $c \equiv \cos \delta$ and $s \equiv \sin \delta$. Finally, the loop function $K=K\left(M_{1}, M_{2}\right)$ is given by

$$
\begin{aligned}
K\left(M_{1}, M_{2}\right) \equiv & -\frac{M_{1}^{2}+4 M_{1} M_{2}+M_{2}^{2}}{M_{1} M_{2}\left(M_{1}+M_{2}\right)} \\
& +\frac{4\left(M_{1}^{2}+M_{1} M_{2}+M_{2}^{2}\right)}{\left(M_{1}-M_{2}\right)\left(M_{1}+M_{2}\right)^{2}} \log \frac{M_{1}}{M_{2}} .
\end{aligned}
$$

Concerning the $\theta$ decay into charged leptons, one needs to add the contribution of the $W$-exchange diagram. The main 
phenomenological constraint comes from $\theta \rightarrow e^{+} e^{-}$. Therefore, in our simplified calculation, we identify the active neutrino with the electron neutrino $\nu_{e}$. Then, the additional contribution to the effective coupling of $\theta$ to electrons is given by

$$
\mathcal{L}_{\theta e \bar{e}}^{W}=i\left(\frac{M_{11}}{f} F_{1}-\frac{M_{22}}{f} F_{2}\right) \frac{2 \sqrt{2} G_{F}}{(4 \pi)^{2}} m_{e} \bar{e} \gamma_{5} e \theta,
$$

which carries a relative factor -2 with respect to the $Z$-exchange contribution. This is why the sum of the two contributions in Eqs. (41) and (45) gives $g_{\theta e \bar{e}} / m_{e}=$ $g_{\theta u \bar{u}} / m_{u}=-g_{\theta d \bar{d}} / m_{d}$, consistent with the one-family result in Eq. (39). Also, the functions $F_{1,2}$ are of the order $m_{i}^{2} / M_{j} \sim m_{\nu}$, so the couplings are of the same order as in Eq. (39). Still, cancellations between the various terms are possible, leading to a suppression of the $\theta$ decay width for special flavor structures. This possibility may deserve a future investigation, since an appropriate family symmetry could in principle raise the pNGB lifetime, so that $\theta$ could become a viable DM candidate even for $m_{\theta}>1 \mathrm{GeV}$, a region where freeze-out could lead to the observed relic density. In this paper, we do not invoke a family symmetry for the suppression of $g_{\theta f \bar{f}}$ to happen; rather, we rely on the one-family estimate given in Eq. (39).

Gauge bosons.-The last effective coupling of the pNGB that may have important phenomenological consequences is the coupling to photons. Let us first discuss the limit of exact global symmetry and then comment on the effect of explicit breaking. In general, the couplings of a NGB to gauge bosons are controlled by the gauge anomalies of the associated global symmetry. In the case that $\theta$ is the Majoron, the global symmetry can be identified with $B-L$, because $\theta$ can be rotated away from the Yukawa interactions by rephasing all the SM fermions with a $B-L$ transformation. Since $B-L$ is anomaly free with respect to the SM gauge symmetries, this rephasing does not generate anomalous couplings of the type $\theta F \tilde{F}$; rather, the only leftover interaction is derivative, $(\theta / f) \partial_{\mu} J_{B-L}^{\mu}$, which is generated by the redefinition of the fermion kinetic terms. One concludes that the Majoron has no anomalous couplings to gauge bosons [23].

This discussion is easily generalized to the $U(1)_{X}$ symmetry that we consider in this paper. First, note that the sterile-neutrino charges are irrelevant for gauge anomalies. In order to allow for fermion masses, we can take $X\left(l_{\alpha}\right)=$ $-X\left(e_{\alpha}^{c}\right)=x_{\alpha}$ for $\alpha=e, \mu, \tau$, and zero $X$ charge for the quarks. Then it is easy to check that $U(1)_{X}$ is anomaly free with respect to electromagnetism and color, and thus $\theta$ does not couple to photons and gluons. The anomaly with EW interactions is proportional to $\sum_{\alpha} x_{\alpha}$ and can also be taken equal to zero for simplicity. In summary, in the limit of exact symmetry, our NGB does not couple to gauge bosons.

It is far more difficult to compute the pNGB couplings to gauge bosons in the presence of explicit-symmetry- breaking sources. One expects that such couplings will arise at some level, since there are no symmetry arguments to prevent them at all orders. However, the computation of the lowest-order nonvanishing contribution is nontrivial. We have shown explicitly above that, in the present framework, $\theta$ couples at tree level to neutrinos only, while, at one-loop, couplings are induced to the Higgs boson as well as to charged fermions. At two-loop, there are a number of diagrams that connect $\theta$ to two-gauge bosons. These diagrams do not necessarily add up to zero: On the one hand, they involve the neutrino-mass parameters that break $U(1)_{X}$ explicitly; thus, they are not expected to respect the anomaly argument above. On the other hand, however, these parameters may not be sufficient to induce an operator $\theta F \tilde{F}$ already at two-loop order.

The computation of these two-loop diagrams is beyond the purpose of the present paper because we will show that a decay $\theta \rightarrow \gamma \gamma$ induced at this order would be irrelevant for phenomenology anyway: Once the constraints from $\theta \rightarrow f \bar{f}$ are imposed, the surviving DM-parameter space is not further reduced by constraints on photons. To see this picture, we estimate the size of a two-loop contribution by taking the effective one-loop couplings in Eq. (39) and computing the usual fermion triangle diagrams with two final-state photons. The effective Lagrangian can be written as

$$
\mathcal{L}_{\theta \gamma \gamma}=\frac{1}{8} g_{\theta \gamma \gamma} \theta \epsilon_{\mu \nu \alpha \beta} F^{\mu \nu} F^{\alpha \beta},
$$

and the corresponding decay width is

$$
\begin{aligned}
& \Gamma(\theta \rightarrow \gamma \gamma)=\frac{1}{64 \pi} g_{\theta \gamma \gamma}^{2} m_{\theta}^{3}, \\
& g_{\theta \gamma \gamma}^{\text {estimate }} \simeq \frac{\alpha}{\pi} \sum_{f} \frac{g_{\theta f \bar{f}}}{m_{f}} G\left(\frac{m_{\theta}}{2 m_{f}}\right),
\end{aligned}
$$

where $G(x)=\left|\arcsin ^{2} x\right| / x^{2}$ [24]. Note that even in the limit of exact NGB, with $m_{\theta} \ll m_{f}$ and thus $G(x) \approx 1$, the sum does not give zero when the sign in Eq. (39) is taken into account. Therefore, we stress again that this is just a conservative estimate, and the computation of all the two-loop contributions may lead to a further cancellation. Neglecting the mass dependence in $G$, the requirement $\Gamma(\theta \rightarrow \gamma \gamma)<1 / \tau_{0}$ gives the order-of-magnitude constraint $g \leqq 4 \times 10^{3}\left(\mathrm{MeV} / m_{\theta}\right)^{3 / 2}\left(\mathrm{eV} / m_{\nu}\right)$. This bound is weaker than the one obtained below Eq. (40) from $\Gamma(\theta \rightarrow f \bar{f})$, as long as $m_{\theta} \lesssim 1 \mathrm{GeV}$, which is the region relevant in the present scenario.

\section{B. Cosmological and astrophysical bounds on $\theta$ couplings}

In practice, the DM lifetime has to be larger than the lifetime of the Universe, because late DM decays affect several cosmological and astrophysical observations. To derive the corresponding bounds on our scenario, we make the assumption that $\theta$ is all the DM in the Universe. For the 
sake of simplicity, here, too, we take the approximation of one lepton family, barring large cancellations between the flavor parameters. A sub-GeV particle could decay into neutrinos, electrons, and photons. We discuss these decay channels in turn.

Let us first consider the decay $\theta \rightarrow \nu \nu$. As was noted in Ref. [5], such a decay could affect the expansion history of the Universe because it represents energy transfer from a nonrelativistic $\theta$ to relativistic neutrinos. Using type Ia supernovae and cosmic microwave background data, one obtains the bound [25]

$$
\Gamma(\theta \rightarrow \nu \nu)<4.5 \times 10^{-20} \mathrm{~s}^{-1} \quad(\text { or } \tau>700 \mathrm{Gyr}) .
$$

Using Eq. (37), the corresponding bound on the coupling of $\theta$ to neutrinos is

$$
g_{\theta \nu \nu}<4 \times 10^{-20}\left(\frac{\mathrm{MeV}}{m_{\theta}}\right)^{1 / 2} .
$$

This upper bound can be improved in the range $30 \mathrm{MeV}<$ $m_{\theta}<200 \mathrm{MeV}$ [26], using searches for the diffuse-neutrino-supernova background by the Super-Kamiokande detector. For masses $m_{\theta}>200 \mathrm{MeV}$, the best limit comes from atmospheric neutrino observations. Since the observed spectrum coincides with theoretical estimates, one can set an upper bound on $g_{\theta \nu \nu}[26,27]$. We use the relation in Eq. (38) to translate these constraints in the $m_{\theta^{-}} g$ plane. The blue region shown in Figs. 3 and 4 is excluded by the combination of the observational bounds discussed above.

Let us now consider the $\theta \rightarrow e^{+} e^{-}$decay. We adapt to our case the analysis performed in Ref. [27]. For $m_{\theta} \leq$ $20 \mathrm{MeV}$, the dominant physical process which constrains the parameter space is annihilation at rest, contributing to the $511 \mathrm{keV}$ line. The limit is approximately given by

$$
\left(\frac{\mathrm{MeV}}{m_{\theta}}\right) \Gamma\left(\theta \rightarrow e^{-} e^{+}\right)<5 \times 10^{-27} \mathrm{~s}^{-1} .
$$

Using Eq. (40), this limit leads to

$$
g_{\theta e e}<9 \times 10^{-24}\left(1-\frac{4 m_{e}^{2}}{m_{\theta}^{2}}\right)^{-1 / 2} .
$$

For $20 \mathrm{MeV} \leq m_{\theta} \leq 1 \mathrm{GeV}$, the dominant process is internal bremsstrahlung, i.e., photons that are radiated from the final electron or positron. One gets a slightly more stringent bound than the one shown in Eq. (50) [27]. The predicted value of the coupling $g_{\theta e e}$ in our model, as follows from Eq. (39), is

$$
g_{\theta e e}=g \frac{G_{F}}{(4 \pi)^{2}} m_{\nu} m_{e}=3 \times 10^{-23}\left(\frac{g}{10^{-3}}\right) \frac{m_{\nu}}{\mathrm{eV}} .
$$

The corresponding observational bound is plotted in Figs. 3 and 4 as the red shaded region.

Note that the value $m_{\theta} \simeq 3 \mathrm{MeV}$, predicted by the Higgs portal, could lead to an excess of $511 \mathrm{keV} \gamma$ rays from the Galactic center of the Milky Way. As discussed in Ref. [28], an approximately $1.5 \mathrm{MeV}$ electron or positron, produced by a $\theta \rightarrow e^{+} e^{-}$decay, is nonrelativistic enough to subsequently annihilate at rest in the Galactic center, leading to a $\gamma$ line at $511 \mathrm{keV}$. In particular, with such a mass one can easily obtain a $\gamma$ flux excess of the order of the one observed by the International Gamma-Ray Astrophysics Laboratory (INTEGRAL) observatory $[28,29]$. However, unless the DM galactic profile is much more cuspy than the usually considered profiles, a DM decay gives a flux that is not sufficiently peaked around the Galactic center $[28,30]$ to be able to reproduce the morphology of the signal observed by INTEGRAL.

We now discuss the decay $\theta \rightarrow \gamma \gamma$. For small masses, $m_{\theta} \lesssim \mathrm{keV}$, the photon's energy is absorbed by the baryonic gas in the early Universe, and the processes of recombination and reionization are affected. One can use the analysis done in Ref. [31] (see also [32]) to bound the coupling $g_{\theta \gamma \gamma}$. For larger masses, $m_{\theta} \gtrsim \mathrm{keV}$, photons are no longer absorbed and they propagate freely. Their contribution to the isotropic diffuse photon background also allows one to bound $g_{\theta \gamma \gamma}[32,33]$.

For the region we are most interested in, stronger bounds can be obtained from the gamma-ray line emission limits from the Milky-Way central region. Indeed, in the range $40 \mathrm{keV}<m_{\theta}<16 \mathrm{MeV}$ [33], one obtains

$$
\Gamma(\theta \rightarrow \gamma \gamma)<10^{-28} \mathrm{~s}^{-1}\left(\frac{m_{\theta}}{\mathrm{MeV}}\right) .
$$

Using Eq. (47), this corresponds to

$$
g_{\theta \gamma \gamma}<4 \times 10^{-21}\left(\frac{\mathrm{MeV}}{m_{\theta}}\right) \mathrm{GeV}^{-1} .
$$

Taking the estimate for $g_{\theta \gamma \gamma}$ given in Eq. (47), all these constraints translate into an upper bound on $g$, which is shown in Figs. 3 and 4 by the brown dash-dotted curve.

We should mention that there are astrophysical constraints on the couplings $g_{\theta \nu \nu}$ [34], $g_{\theta e e}$ [35], and $g_{\theta \gamma \gamma}$ [35,36], based on stellar energy loss due to $\theta$ emission, provided that $m_{\theta}$ is low enough to be produced in stellar interiors. These limits are valid without the need to assume that $\theta$ is DM. However, these astrophysical limits are, in general, weaker than the ones mentioned above.

We would also like to note that the region we consider for pNGB-DM masses includes the keV scale, corresponding to warm dark matter (WDM). Since, at the epoch of structure formation, WDM has free-streaming lengths below the Mpc scale, having WDM at least as a nonnegligible DM component can alleviate some of the disagreement between the standard cold-DM scenario and a variety of galactic observations at small scales [37]. The ideal observation which could place a limit on WDM is the Lyman- $\alpha$ forest, i.e., the Lyman- $\alpha$ absorption produced by intervening neutral hydrogen in the spectra of light emitted by distant quasars. Using Lyman- $\alpha$ observations together with other cosmological data sets, different groups have put lower limits on $m_{\mathrm{WDM}}$, assuming that WDM has a thermal distribution and that it is the whole of DM. The bounds in the literature [38] differ by factors of a few, 
ranging from $0.5 \mathrm{keV}$ up to $4 \mathrm{keV}$. In our case, the DM species is not in thermal equilibrium, and the bounds can change slightly compared to the WDM thermal relic case. In addition, if WDM is only a part of the whole of DM, the bounds are relaxed. We should mention that there are some potential problems in obtaining these bounds, and they should be regarded as controversial. On the one hand, there could be large systematic errors, and, on the other hand, the Lyman- $\alpha$ analysis has to be performed at scales that are already in the nonlinear regime, where calculations are less reliable. If one disposes of the Lyman- $\alpha$ data, these bounds on WDM disappear altogether [39].

\section{CONCLUSIONS}

We have proposed a new pseudoscalar gauge-singlet $\mathrm{DM}$ candidate $\theta$ with mass in the $\mathrm{keV}-\mathrm{MeV}$ range. Its couplings to the SM particles are feeble, because they are mediated by new physics at a large scale $f$, which we identify with the seesaw scale. The $\theta$ relic density can be produced by the scattering with the heavy particles (the sterile neutrinos), at temperatures of the order of $f$, or, alternatively, it can be generated at the EW scale through the Higgs portal by the tiny $\theta-H$ coupling $\lambda \sim 10^{-10}$, which is induced by the seesaw interactions. Today, $\theta$ decays into light neutrinos and, if it is heavier than $1 \mathrm{MeV}$, into $e^{+} e^{-}$, with rates that can saturate the present upper bounds.

We have argued that such a candidate is theoretically well motivated. The heavy new physics sector is generically associated with several global symmetries. Some of the corresponding pNGBs may remain light if the explicitsymmetry-breaking effects are sufficiently small. For concreteness, we have demonstrated that a $U(1)_{X}$ family symmetry of the sterile-neutrino sector can be broken collectively by a set of neutrino Yukawa couplings, so that the pNGB mass $m_{\theta}$ is proportional to the EW scale and to the (small) product of the Yukawa couplings. In such a scenario, $m_{\theta}$ is not quadratically sensitive to the cutoff (at leading order); therefore, the presence of a light DM scalar below the EW scale is justified.

In order to calculate the $\theta$ relic density, we have computed the rate of the $\theta$ interactions with a sterile neutrino $N$ and, through the Higgs portal, with the SM particles, and we have studied the Boltzmann equation numerically for the $\theta$ number density. In this framework, there are only two independent parameters: the mass of the pNGB, $m_{\theta}$, and the coupling of the pNGB to the sterile neutrino, $g$. The fact that there are only two independent parameters makes our scenario especially predictive. We find that the Higgs portal produces the desired DM relic density, through the freeze-in mechanism, for a unique value of the DM mass, $m_{\theta} \simeq 3 \mathrm{MeV}$. This prediction relies only on the relation between the DM mass and its coupling to the Higgs, $m_{\theta}^{2}=$ $\lambda v^{2}$, which is well justified in the case of our pNGB. As long as the reheating temperature is sufficiently high, the sterile-neutrino portal can produce the required relic density by freeze-in for smaller values of $m_{\theta}$, from $\simeq 3 \mathrm{MeV}$ down to $0.15 \mathrm{keV}$. These results are summarized in Fig. 1.

The constraints from $\Gamma(\theta \rightarrow \nu \nu)$, however, exclude the region $m_{\theta} \lesssim 1(100) \mathrm{keV}$, for a light-neutrino mass $m_{\nu}=$ $0.05(1) \mathrm{eV}$. In addition, the constraints from $\Gamma\left(\theta \rightarrow e^{+} e^{-}\right)$ put an upper bound on the coupling $g$ in the region $m_{\theta} \gtrsim$ $1 \mathrm{MeV}$ : For $m_{\nu}=0.05(1) \mathrm{eV}$ and $g \sim 10^{-3}\left(10^{-4}\right)$, the expected electron-positron flux is close to the present sensitivity. These results are summarized in Figs. 3 and 4.

In turn, the seesaw scale can be constrained, by requiring our pNGB to be a viable DM candidate in agreement with all the bounds above. In the case of freeze-in through the sterile-neutrino portal, we find $10^{5} \mathrm{GeV} \lesssim m_{N} \lesssim$ $10^{10} \mathrm{GeV}$, and a SSB scale $f \sim 10^{3} \mathrm{~m}_{N}$. In the case of the Higgs portal, one has instead $10^{10} \mathrm{GeV} \lesssim m_{N} \lesssim$ $10^{14} \mathrm{GeV}$, and, correspondingly, $10^{13} \mathrm{GeV} \lesssim f \lesssim M_{P}$.

Finally, we note that our analysis of the parameter space was mostly performed in the one-family approximation, that is, neglecting possible hierarchies among the sterileneutrino-mass parameters and the neutrino Dirac Yukawa couplings. Some of our main results are independent from this approximation, such as the allowed range for the DM mass. On the contrary, the bounds on $g$ and $m_{N}$ are obviously sensitive to the flavor structure. A rough idea of these dependencies can be grasped by comparing the case $m_{\nu}=0.05 \mathrm{eV}$ (Fig. 3) with the case $m_{\nu}=1 \mathrm{eV}$ (Fig. 4). A more detailed exploration of the flavor-parameter space of this scenario is desirable, in particular, if one wants to compare the results with neutrino flavor models.

\section{ACKNOWLEDGMENTS}

It is a pleasure to thank K.S. Babu, X. Chu, J.R. Espinosa, D. Greynat, K. Jedamzik, S. Lavignac, S. Peris, S. Palomares-Ruiz, A. Pomarol, M. Quiros, J. Redondo, J. Serra, M. H. G. Tytgat, and A. Varagnolo for very useful discussions. The work of M. F. was supported in part by the Spanish Research Project No. CICYTFEDER-FPA-2008-01430, the Consolider-Ingenio 2010 Programme CPAN No. (CS-D2007-00042), and the Marie-Curie Reintegration Grant No. PERG06-GA-2009256374 within the European Community Seventh Framework Programme (FP7). M.F. also thanks the Service de Physique Théorique of the Université Libre de Bruxelles for hospitality. The work of T. H. is supported by the FNRS-FRS, the IISN, and the Belgian Science Policy (IAP VI-11). T. H. thanks J. R. Espinosa and the IFAE at Barcelona for hospitality. The work of E.M. has been partly supported by Contracts No. CICYT-FEDERFPA2008-01430 and No. 2009SGR894.

[1] Y. Chikashige, R. N. Mohapatra, and R. D. Peccei, Are There Real Goldstone Bosons Associated with Broken Lepton Number?, Phys. Lett. B 98, 265 (1981). 
[2] E. K. Akhmedov, Z. G. Berezhiani, G. Senjanovic, and Z.j. Tao, Planck Scale Effects in Neutrino Physics, Phys. Rev. D 47, 3245 (1993).

[3] I.Z. Rothstein, K. S. Babu, and D. Seckel, Planck Scale Symmetry Breaking and Majoron Physics, Nucl. Phys. B403, 725 (1993).

[4] V. Berezinsky and J. W. F. Valle, The Kev Majoron as a Dark Matter Particle, Phys. Lett. B 318, 360 (1993).

[5] M. Lattanzi and J.W.F. Valle, Decaying Warm Dark Matter and Neutrino Masses, Phys. Rev. Lett. 99, 121301 (2007).

[6] F. Bazzocchi, M. Lattanzi, S. Riemer-Sorensen, and J.W.F. Valle, X-Ray Photons from Late-Decaying Majoron Dark Matter, J. Cosmol. Astropart. Phys. 08 (2008) 013; J. N. Esteves, F. R. Joaquim, A. S. Joshipura, J. C. Romao, M. A. Tortola, and J.W.F. Valle, $A_{4}$-Based Neutrino Masses with Majoron Decaying Dark Matter, Phys. Rev. D 82, 073008 (2010).

[7] P. H. Gu, E. Ma, and U. Sarkar, Pseudo-Majoron as Dark Matter, Phys. Lett. B 690, 145 (2010).

[8] L. J. Hall, K. Jedamzik, J. March-Russell, and S. M. West, Freeze-In Production of FIMP Dark Matter, J. High Energy Phys. 03 (2010) 080.

[9] C. T. Hill and G. G. Ross, Pseudogoldstone Bosons and New Macroscopic Forces, Phys. Lett. B 203, 125 (1988); Models and New Phenomenological Implications of a Class of Pseudogoldstone Bosons, Nucl. Phys. B311, 253 (1988).

[10] M. Schmaltz and D. Tucker-Smith, Little Higgs Review, Annu. Rev. Nucl. Part. Sci. 55, 229 (2005); M. Perelstein, Little Higgs Models and Their Phenomenology, Prog. Part. Nucl. Phys. 58, 247 (2007).

[11] Notice that Eq. (6) is the condition for DM to thermalize with the SM sector at $T \simeq m_{h}$ (where the rates reach the maximum; see Fig. 2.) This condition is different from the one usually considered for the freeze-out scenario, where one requires that the scattering processes are in thermal equilibrium at $T \lesssim m_{\theta}$. This condition leads to a value of $\lambda$ about 2 orders of magnitude larger than the one in Eq. (6).

[12] In Eqs. (9)-(13), we included the usual initial-state spinand color-average factor, as well as the final-state identical-particle factor $1 / 2$. In $\sigma(h h \rightarrow \theta \theta)$, we neglected subleading terms of order $\lambda^{3}$ and $\lambda^{4}$ (which are displayed, e.g., in Ref. [13]).

[13] W. L. Guo and Y. L. Wu, The Real Singlet Scalar Dark Matter Model, J. High Energy Phys. 10 (2010) 083.

[14] M. Farina, D. Pappadopulo, and A. Strumia, CDMS Stands for Constrained Dark Matter Singlet, Phys. Lett. B 688, 329 (2010).

[15] Actually, if one takes the value of $\gamma_{D h} /(H s)$ at its maximum value, i.e., at $T \sim m_{h} / 3.5$, one obtains a number density equal to $70 \%$ of the exact result. Therefore, the production of a particle through freeze-in of a decay is essentially given by the maximum value of the corresponding thermalization rate. Note also that if one takes instead $N_{\theta} n_{h}^{\mathrm{eq}} \Gamma(h \rightarrow \theta \theta) /$ (Hs) at its maximum value, which occurs for $T \sim m_{h} / 3.0$, it turns out that one gets an even better approximation, as this overestimates the exact result by only $3 \%$.
[16] It is well known that, if a sterile neutrino has Yukawa couplings inducing a light-neutrino mass of the order of the atmospheric- or solar-neutrino-mass scales, the decays/inverse decays of the sterile neutrino are in thermal equilibrium at $T \sim m_{N}$. Various cosmological issues can put constraints on thermal sterile neutrinos; see, e.g., [17]. However, they become relevant for sterile-neutrino masses below about the GeV scale, which are not pertinent in our case; see Figs. 3 and 4.

[17] A. Y. Smirnov and R. Zukanovich Funchal, Sterile Neutrinos: Direct Mixing Effects versus Induced Mass Matrix of Active Neutrinos, Phys. Rev. D 74, 013001 (2006).

[18] P. Sikivie, Axion Cosmology, Lect. Notes Phys. 741, 19 (2008).

[19] R. Barbieri, L. J. Hall, S. J. Oliver, and A. Strumia, Dark Energy and Right-Handed Neutrinos, Phys. Lett. B 625, 189 (2005).

[20] The choice of $X$ charges is made to realize a model of type (iii), in accordance with the classification of the previous section. This choice is not unique. An equivalent possibility is a $U(1)_{X}$ symmetry with charges $X\left(\nu_{1}^{c}\right)=-1$, $X\left(\nu_{2}^{c}\right)=1, X\left(l_{\alpha}\right)=0$, broken spontaneously by a scalar field of charge $X(\phi)=1$. Then in Eq. (33) one should replace $m_{\alpha 1} \rightarrow m_{\alpha 1} e^{i \theta / f}$ and $m_{\alpha 2} \rightarrow m_{\alpha 2} e^{-i \theta / f}$, while the entries $M_{11}$ and $M_{22}$ are independent from $\theta$ and represent the source of explicit symmetry breaking. It is easy to check that the two realizations are equivalent, since the Lagrangian is the same up to a rephasing of the neutrino fields.

[21] Note that the field $\theta$ can be rotated away from Eq. (33) by phase redefinitions, as long as the $n_{f}$ entries that explicitly break $U(1)_{X}$ are set to zero. This is slightly different from the general case discussed following Eq. (29), where $n_{f}+$ 1 entries must vanish, for the trivial reason that here the same phase field appears (with opposite sign) in two independent entries. The only physically relevant fact still holds: The product of four independent entries is needed to generate a mass for $\theta$.

[22] A. Pilaftsis, Astrophysical and Terrestrial Constraints on Singlet Majoron Models, Phys. Rev. D 49, 2398 (1994).

[23] Equivalently, one may remove $\theta$ from the Yukawa interactions by an $L$ transformation; in this case, one is left with the couplings $(\theta / f)\left[\partial_{\mu} J_{L}^{\mu}+\left(c_{W} F_{W} \tilde{F}_{W}+c_{Y} F_{Y} \tilde{F}_{Y}\right) /\right.$ $\left.\left(16 \pi^{2}\right)\right]$, where $c_{W, Y} \neq 0$ account for the EW anomalies of the lepton-number symmetry. There is no physical difference between the two pictures. In particular, one can check that (i) $\theta$ does not couple to weak gauge bosons at one-loop even in the $L$ case, because the triangle diagrams generated by the derivative coupling to $J_{L}^{\mu}$ cancel $c_{W, Y}$ exactly; (ii) $\theta$ does not decay to two on-shell quarks even in the $B-L$ case, because the quarks are vectorlike under $B-L$.

[24] L. Ametller, L. Bergstrom, A. Bramon, and E. Masso, The Quark Triangle: Application to Pion and Eta Decays, Nucl. Phys. B228, 301 (1983).

[25] Y. Gong and X. Chen, Cosmological Constraints on Invisible Decay of Dark Matter, Phys. Rev. D 77, 103511 (2008).

[26] S. Palomares-Ruiz, Model-Independent Bound on the Dark Matter Lifetime, Phys. Lett. B 665, 50 (2008). 
[27] N. F. Bell, A. J. Galea, and K. Petraki, Lifetime Constraints for Late Dark Matter Decay, Phys. Rev. D 82, 023514 (2010).

[28] N. Prantzos, C. Boehm, A. M. Bykov, R. Diehl, K. Ferrière, N. Guessoum, P. Jean, J. Knoedlseder, A. Marcowith, I. V. Moskalenko, A. Strong, and G. Weidenspointner, The $511 \mathrm{keV}$ Emission from Positron Annihilation in the Galaxy, Rev. Mod. Phys. 83, 1001 (2011) and references therein.

[29] G. Weidenspointner, G. Skinner, P. Jean, J. Knödlseder, P. von Ballmoos, G. Bignami, R. Diehl, A. W. Strong, B. Cordier, S. Schanne, and C. Winkler, An Asymmetric Distribution of Positrons in the Galactic Disk Revealed by Gamma-Rays, Nature (London) 451, 159 (2008).

[30] Y. Ascasibar, P. Jean, C. Boehm, and J. Knoedlseder, Constraints on Dark Matter and the Shape of the Milky Way Dark Halo from the $511 \mathrm{keV}$ Line, Mon. Not. R. Astron. Soc. 368, 1695 (2006).

[31] L. Zhang, X. Chen, M. Kamionkowski, Z.g. Si, and Z. Zheng, Contraints on Radiative Dark-Matter Decay from the Cosmic Microwave Background, Phys. Rev. D 76, 061301 (2007).

[32] X.L. Chen and M. Kamionkowski, Particle Decays During the Cosmic Dark Ages, Phys. Rev. D 70, 043502 (2004).

[33] H. Yuksel and M. D. Kistler, Dark Matter Might Decay... Just Not Today!, Phys. Rev. D 78, 023502 (2008).

[34] J. A. Grifols, E. Masso, and S. Peris, Majoron Couplings to Neutrinos and SN1987A, Phys. Lett. B 215, 593 (1988).

[35] G. G. Raffelt, Stars as Laboratories for Fundamental Physics: The Astrophysics of Neutrinos, Axions, and
Other Weakly Interacting Particles (University of Chicago,, Chicago, 1996), p. 664.

[36] E. Masso and R. Toldra, On a Light Spinless Particle Coupled to Photons, Phys. Rev. D 52, 1755 (1995).

[37] B. Moore, S. Ghigna, F. Governato, G. Lake, T. R. Quinn, J. Stadel, and P. Tozzi, Dark Matter Substructure within Galactic Halos, Astrophys. J. 524, L19 (1999); G. Gentile, A. Burkert, P. Salucci, U. Klein, and F. Walter, The Dwarf Galaxy DDO 47: Testing Cusps Hiding in Triaxial Halos, Astrophys. J. 634, L145 (2005).

[38] M. Viel, J. Lesgourgues, M. G. Haehnelt, S. Matarrese, and A. Riotto, Constraining Warm Dark Matter Candidates Including Sterile Neutrinos and Light Gravitinos with WMAP and the Lyman-Alpha Forest, Phys. Rev. D 71, 063534 (2005); K. Abazajian, Linear Cosmological Structure Limits on Warm Dark Matter, Phys. Rev. D 73, 063513 (2006); M. Viel, J. Lesgourgues, M. G. Haehnelt, S. Matarrese, and A. Riotto, Can Sterile Neutrinos Be Ruled Out as Warm Dark Matter Candidates?, Phys. Rev. Lett. 97, 071301 (2006); U. Seljak, A. Makarov, P. McDonald, and H. Trac, Can Sterile Neutrinos Be the Dark Matter?, Phys. Rev. Lett. 97, 191303 (2006); M. Viel, G. D. Becker, J. S. Bolton, M. G. Haehnelt, M. Rauch, and W. L.W. Sargent, How Cold Is Cold Dark Matter? Small Scales Constraints from the Flux Power Spectrum of the HighRedshift Lyman-Alpha Forest, Phys. Rev. Lett. 100, 041304 (2008).

[39] S. Hannestad and G. Raffelt, Cosmological Mass Limits on Neutrinos, Axions, and Other Light Particles, J. Cosmol. Astropart. Phys. 04 (2004) 008. 